\title{
Assessing hydrocarbon prospects in Abu Madi formation using well logging data in El-Qara field, Nile Delta Basin, Egypt
}

\author{
Mohammad Abdelfattah Sarhan ${ }^{1}$ (D)
}

Received: 18 March 2021 / Accepted: 8 June 2021 / Published online: 26 June 2021

(c) The Author(s) 2021

\begin{abstract}
In this work, the petrophysical properties of Abu Madi reservoir in El-Qara Field at northern Nile Delta Basin (NDB) were evaluated depending on well logging data of two wells: El-Qara-2 and El-Qara-3. This evaluation revealed that in El-Qara-2 well, the promising gas zone is detected between depths of 3315 and $3358 \mathrm{~m}$, while in El-Qara-3 well, the best gas interval is detected between depths of 3358 and $3371 \mathrm{~m}$. In addition to the production test parameters (gas rate, condensate rate, gas gravity, condensate gravity, gas-to-oil ratio, flowing tubing head pressure, flowing bottom hole pressure, and static bottom hole pressure), the calculated petrophysical parameters (shale volume, total porosity, effective porosity, and water saturation) for both intervals were relatively similar. This confirms that the investigated wells were drilled at the same reservoir interval within Abu Madi Fm. The depth variation in the examined zones was attributed to the presence of buried normal faults between El-Qara-2 and El-Qara-3 wells. This observation may be supported from the tectonic influence during the deposition of Abu Madi Fm. as a portion of the Messinian syn-rift megasequence beneath the NDB.
\end{abstract}

Keywords Well logging $\cdot$ Abu Madi Fm. $\cdot$ El-Qara field $\cdot$ Nile Delta basin

\section{Introduction}

Nile Delta Basin (NDB) is a passive-margin basin occupying approximately $250,000 \mathrm{~km}^{2}$ in the eastern Mediterranean area. It was formed because of the thermal subsidence consequent of the extensional tectonic phase, which isolated the African plate away from the Eurasian plate during the Late Triassic-Early Cretaceous age (Dolson et al. 2001; May 1991).

In the last five decades, several trillion cubic feet of gas have been explored in the NDB and found in various stratigraphic levels extending from the Oligocene to the Pliocene. Since 1963, the Messinian Abu Madi sandstones have been the primary source of gas and condensate for several petroleum companies in both onshore and offshore parts of the NDB (Abu El-Ella 1990).

During the Messinian age, the Mediterranean Sea was confined from the global ocean and dried owing to extraordinary evaporation. This process induced a major

Mohammad Abdelfattah Sarhan

msarhan@du.edu.eg

1 Geology Department, Faculty of Science, Damietta University, New Damietta City 34517, Egypt unconformity surface and led to the deposition of thick evaporite sections (Rosetta Fm.). In this way, a north-trending fluvial system (The Eonile of Said 1990) was formed at the latitude of Cairo (Harms and Wary 1990). This Eonile formed a deep canyon incision when the drainage system changed in the Early Miocene from northwest (from the Qattara Depression) to northward (to its current position in the Mediterranean Sea) during the Messinian age owing to the eastward tilting of the land (Ross and Uchupi 1977). This canyon cut into the pre-existing Cenozoic-Mesozoic section and transported huge quantities of sediments into the Mediterranean Basin (El-Barkooky and Helal 2002).

Abu Madi Fm. consists of sandstone and shale intercalations (Rizzini et al. 1976), which was deposited during the Messinian age (Egyptian General Petroleum Corporation (EGPC), 1994; El Heiny and Morsi 1992). The term "Abu Madi Paleovalley" refers to the Proto-Nile distributaries in the northern part of the NDB, which incised the pre-existing shelf and slope of the Mediterranean Sea and formed a wide fluvial system. This incision resulted from a remarkable decrease in the ocean level worldwide during the Messinian age (Harms and Wary 1990; Barber 1981; Sestini 1989). Thus, all the sandstones of Abu Madi Fm. were deposited as incised-valley fills reflecting the lowstand 
system tract that was topped by transgressive estuarine facies (Salem et al. 2005).

El-Qara Field lies within the Abu Madi Paleovalley to the north of NDB close to the Egyptian Coast (Fig. 1). It contains several drilled wells that produce gas from the sandstones of Abu Madi Fm. In this study, two wells in El-Qara Field were examined, namely, El-Qara-2 and ElQara-3. El-Qara-2 well was drilled with a total measured depth of $3460 \mathrm{~m}$ at the intersection between latitude $31^{\circ} 28^{\prime}$ $57.1640^{\prime \prime} \mathrm{N}$ and longitude $31^{\circ} 18^{\prime} 40.8516^{\prime \prime}$ E. Alternatively, El-Qara-3 well has a total measured depth of $3505 \mathrm{~m}$ at the point between latitude $31^{\circ} 29^{\prime} 34.91^{\prime \prime} \mathrm{N}$ and longitude $31^{\circ} 16^{\prime}$ 54.641"E (Fig. 1).

Herein, the petrophysical properties of the reservoir intervals within the sandstones of Abu Madi Fm. in El-Qara-2 and El-Qara-3 wells were assessed to examine the efficiency of these intervals as gas-bearing resources in El-Qara Field in NDB.

\section{Geological setting}

NDB exhibits a thick sedimentary section from the Tertiary to Quaternary ages. Throughout the Jurassic-Early Cretaceous, a major faulted and generally flexed zone formed in the northern Egypt, called the "Hinge Zone" (Said 1962; Sestini 1989). This zone separates the northern Egypt on an east-west trend (Mosconi et al. 1996), characterizing the southern boundary of the rifted continental margin (Bertello et al. 1996) and controlling the stratigraphic and structural evolution of the north-central basins in Egypt (Said 1981; Harms and Wary 1990; Arisi et al. 1994). Numerous extensional faults formed along this Hinge Zone with strong faulting downward to the north resulted in a significant thick sedimentary succession (5000-7000 m) to the north of the hinge line, while only $500-1500 \mathrm{~m}$ of the sedimentary cover was documented to the south (Harms and Wray 1990; Sarhan and Hemdan 1994; EGPC 1994).

The NDB underwent dynamic subsidence throughout the Late Miocene owing to the uplift of Sinai and the Eastern Desert and the start of the Gulf of Suez rift and the Aqaba-Jordan shear (Kamel et al. 1998). However, during the Pliocene-Pleistocene age, a belt of compression was developed, associated with the sinistral wrench movement. This phenomenon led to the creation of the northeast-southwest Pelusium fault, which crosses the northwest seaward parts of Sinai, bounds the southern part of the Nile Delta, and expands to the western part of the African plate (EGPC 1994; Zaghloul et al. 2001).

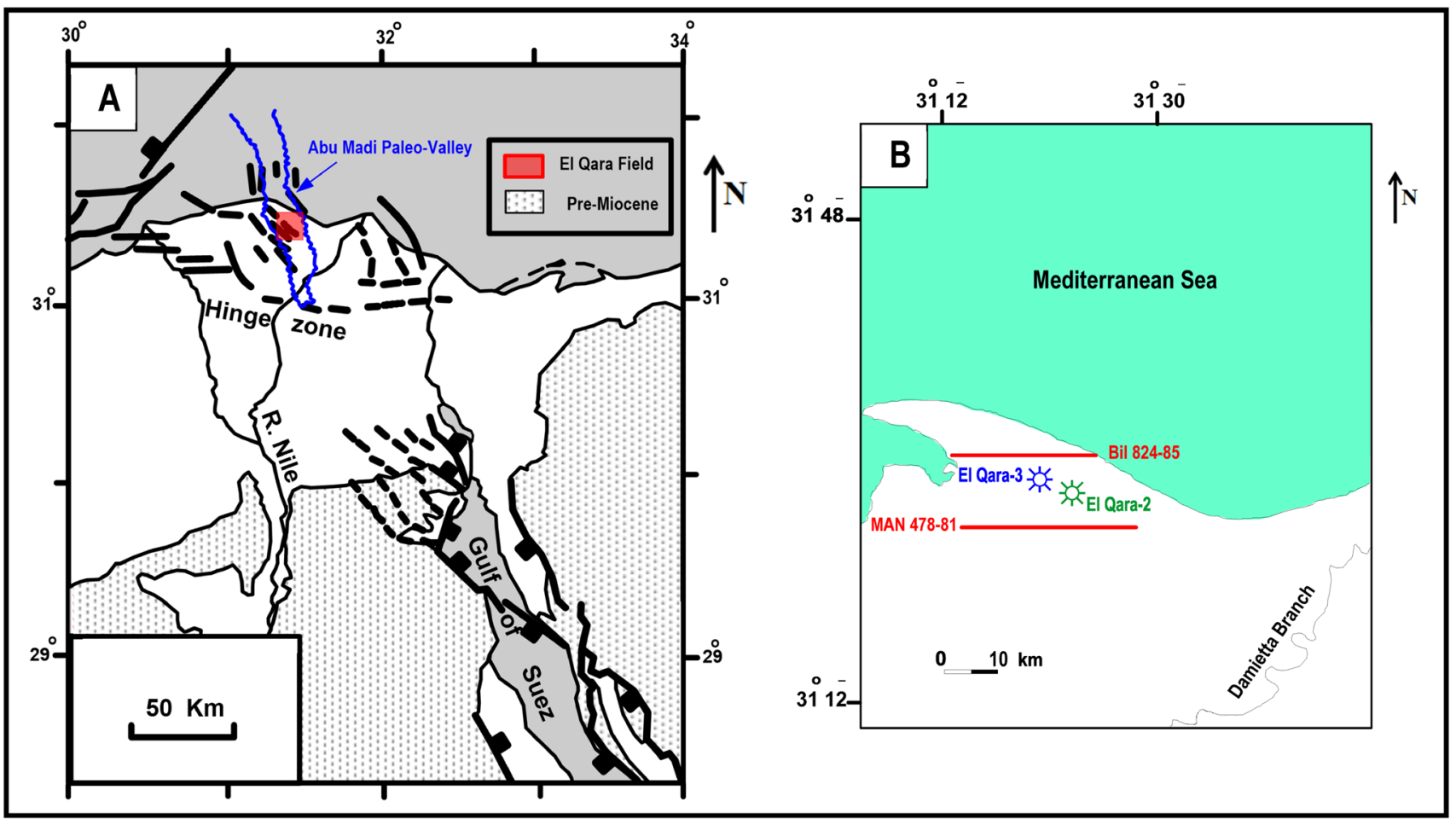

Fig. 1 a Map displays the location of El-Qara Field, northern Nile Delta Basin, and summarizes the active faults within the Abu Madi Paleovalley during the Late Miocene age (Sarhan et al. 2014). b
Locations of El-Qara-2 and El-Qara-3 wells and two interpreted seismic profiles in the examined field 
The Miocene section in the NDB includes the Abu Madi Fm. (or its equivalent Rosetta Fm.) and Qawasim Fm. from top to bottom, characterizing the Messinian age. The Sidi Salim Fm. represents the Middle Miocene age, and the Qantara Fm. represents the Early Miocene age. The Pliocene succession in the NDB includes the Mit Ghamr Fm. and El Wastani Fm. from top to bottom, representing the Late Pliocene age and the Kafr El-Sheikh Fm. of the Early-Middle Pliocene age (El Heiny and Enani 1996; EGPC 1994; Vandre et al. 2007).

Generally, the Abu Madi Fm. in EL-Qara Field comprises sandstones with shale interbeds. Based on the composite logs of EL-Qara-2 well, all the shales are gray, dark gray, firm, sub-blocky, silty grained, and locally to siltstone, calcareous, and locally highly calcareous, and occasionally pyritic. However, the sandstone beds are quartzose, grayish, off white, fine to coarse grained in the basal sand body, fine to medium grained, occasionally coarse grained, moderately to poorly sorted in the middle-upper part, weakly to moderately cemented by calcareous cement, moderately hard to friable, and locally glauconitic (Fig. 2).

According to the composite logs of EL-Qara-3 well, the shale beds are light gray, firm, sub-blocky, silty to very silty grained to siltstone in some parts, and noncalcareous to slightly calcareous and show traces of glauconite. However, the sandstone interbeds are quartzose, colorless, occasionally orange, fine to medium grained, locally coarse grained, subrounded to subangular, moderately sorted, carbonatic cement, and semi friable (Fig. 2).
Fig. 2 Lithostratigraphic column for Abu Madi Fm. based on the available composite logs for El-Qara-2 and El-Qara-3 wells
EI Qara-3 Well

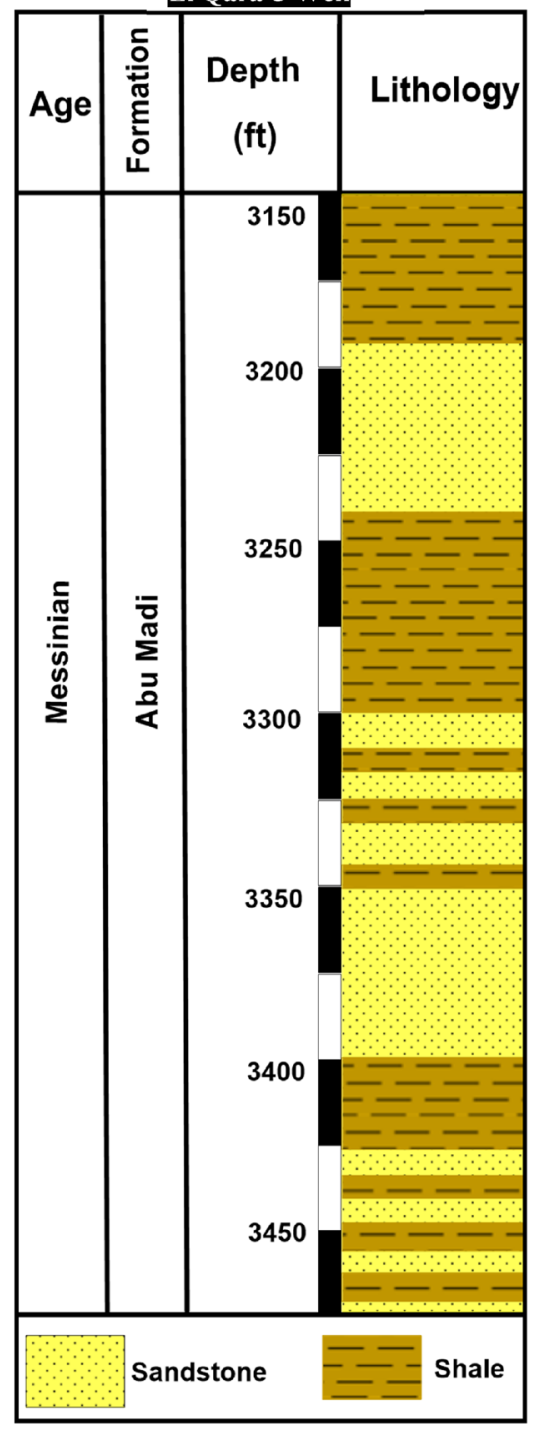

El Qara-2 Well

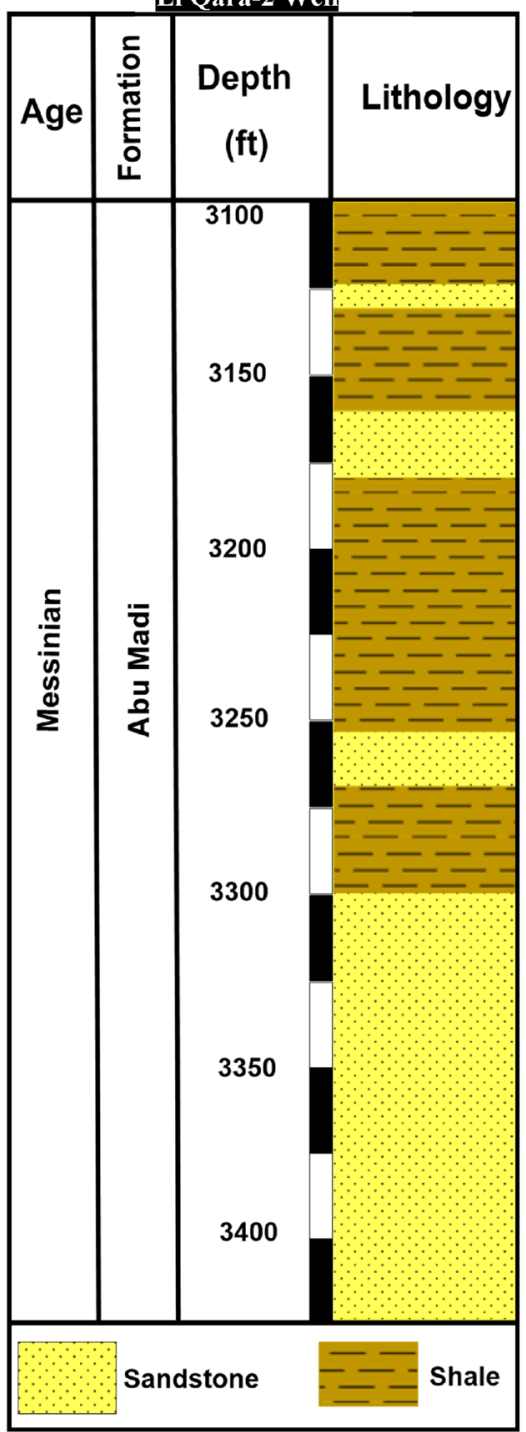




\section{Data and methods}

The hydrocarbon prospects of Abu Madi sandstones in ElQara Field were assessed using Techlog software for the conventional wireline logs of EL-Qara-2 and EL-Qara-3 wells. The log suit of each well includes caliper, gamma ray, sonic, deep resistivity, bulk density, and neutron porosity logs (Figs. 3, 4). The composite logs of EL-Qara-2 and EL-Qara-3 wells were evaluated, and the data of drill stem test (DST) and production tests were also available.

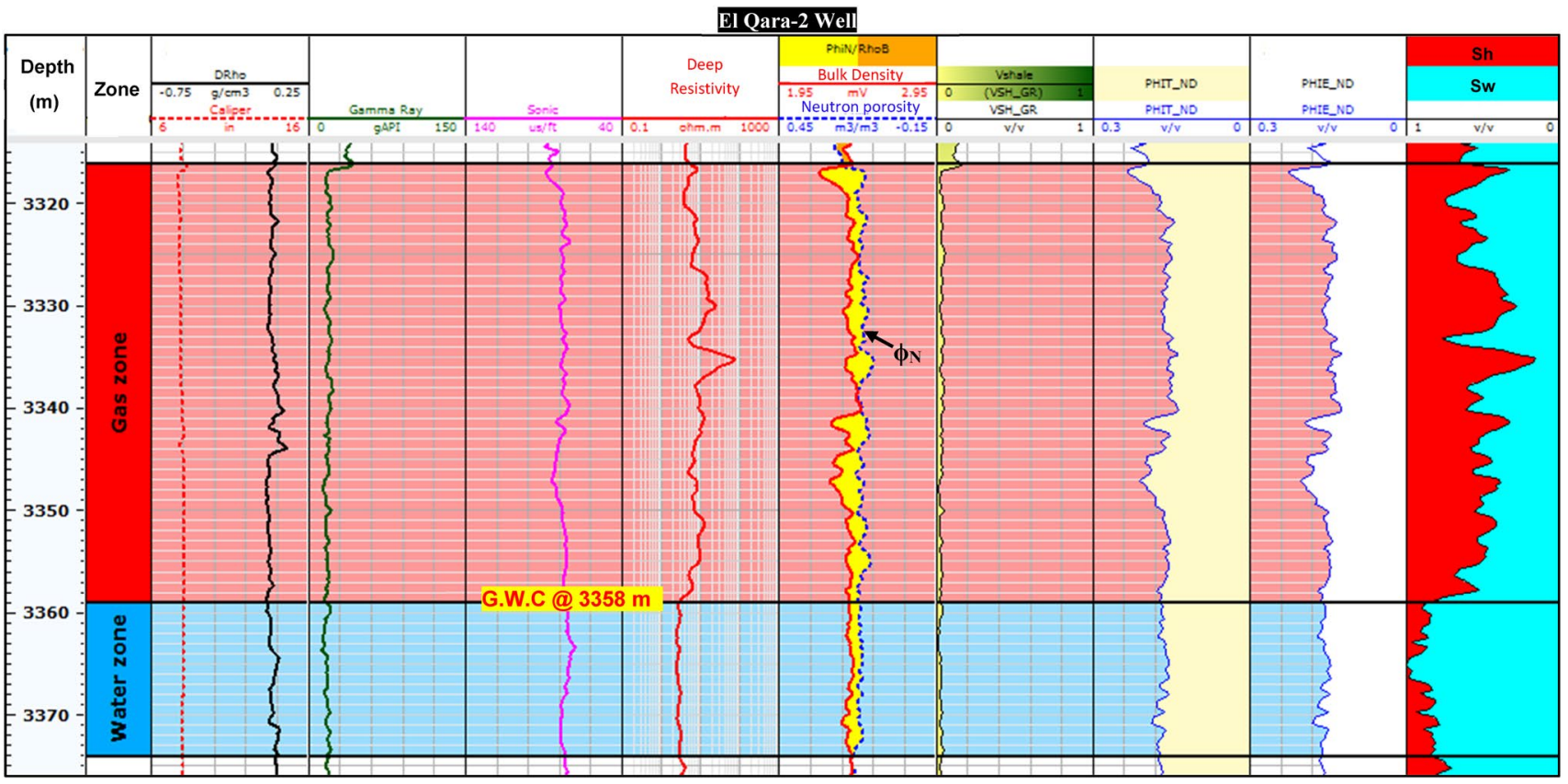

Fig. 3 Wireline log data with an interpretation for the gas zone in El-Qara-2 well

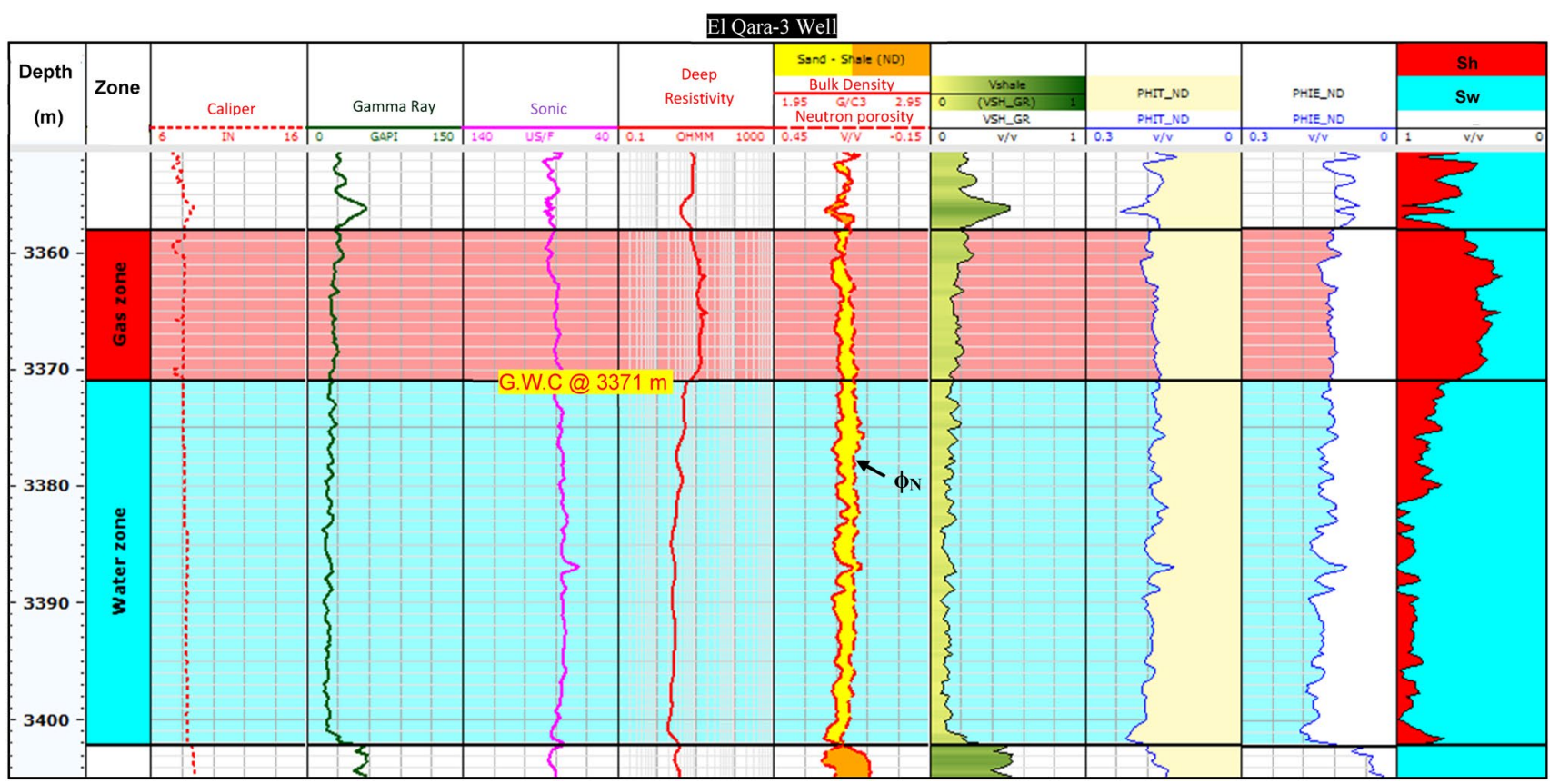

Fig. 4 Wireline log data with an interpretation for the gas interval in El-Qara-3 well 
Table 1 Well log data and output results for gas zone in El-Qara-2 well

\begin{tabular}{|c|c|c|c|c|c|c|c|c|c|}
\hline \multicolumn{6}{|c|}{ Input well log data } & \multicolumn{4}{|l|}{ Output results } \\
\hline Depth (ft) & $\begin{array}{l}\text { Gamma } \\
\text { ray (API) }\end{array}$ & Sonic (US/F) & $\begin{array}{l}\text { Deep } \\
\text { resistivity } \\
\left(\Omega \mathrm{m}^{2} / \mathrm{m}\right)\end{array}$ & $\begin{array}{l}\text { Neutron } \\
\text { porosity }\end{array}$ & Density (G/C3) & Shale volume & Total porosity & $\begin{array}{l}\text { Effective } \\
\text { porosity }\end{array}$ & Water saturation \\
\hline 3315.02 & 36.90 & 80.19 & 3.94 & 0.22 & 2.37 & 0.12 & 0.21 & 0.17 & 0.63 \\
\hline 3315.18 & 35.88 & 82.73 & 3.92 & 0.22 & 2.38 & 0.12 & 0.20 & 0.17 & 0.64 \\
\hline 3315.33 & 34.37 & 85.99 & 4.00 & 0.21 & 2.39 & 0.11 & 0.20 & 0.17 & 0.64 \\
\hline 3315.48 & 34.56 & 87.19 & 4.08 & 0.21 & 2.39 & 0.11 & 0.20 & 0.16 & 0.65 \\
\hline 3315.63 & 36.98 & 86.81 & 4.14 & 0.21 & 2.40 & 0.12 & 0.20 & 0.16 & 0.66 \\
\hline 3315.78 & 39.80 & 86.33 & 4.37 & 0.21 & 2.41 & 0.14 & 0.19 & 0.15 & 0.66 \\
\hline 3315.94 & 41.68 & 85.77 & 4.87 & 0.21 & 2.42 & 0.15 & 0.19 & 0.15 & 0.65 \\
\hline 3316.09 & 42.84 & 84.99 & 5.37 & 0.22 & 2.42 & 0.16 & 0.19 & 0.14 & 0.62 \\
\hline 3316.24 & 42.51 & 84.58 & 5.79 & 0.22 & 2.39 & 0.16 & 0.20 & 0.15 & 0.56 \\
\hline 3316.39 & 38.59 & 85.12 & 6.69 & 0.20 & 2.33 & 0.13 & 0.21 & 0.17 & 0.46 \\
\hline 3316.54 & 31.06 & 86.24 & 8.12 & 0.17 & 2.26 & 0.09 & 0.23 & 0.20 & 0.37 \\
\hline 3316.70 & 23.39 & 87.50 & 8.74 & 0.14 & 2.22 & 0.06 & 0.23 & 0.22 & 0.34 \\
\hline 3316.85 & 18.87 & 88.75 & 7.87 & 0.13 & 2.20 & 0.04 & 0.23 & 0.22 & 0.36 \\
\hline 3317.00 & 17.64 & 89.13 & 6.54 & 0.12 & 2.20 & 0.03 & 0.23 & 0.22 & 0.39 \\
\hline 3317.15 & 17.62 & 88.39 & 5.58 & 0.12 & 2.21 & 0.03 & 0.23 & 0.22 & 0.43 \\
\hline 3317.30 & 17.26 & 87.61 & 5.03 & 0.12 & 2.22 & 0.03 & 0.23 & 0.22 & 0.46 \\
\hline 3317.46 & 16.56 & 87.34 & 4.74 & 0.12 & 2.23 & 0.03 & 0.22 & 0.21 & 0.48 \\
\hline 3317.61 & 16.12 & 86.73 & 4.63 & 0.12 & 2.25 & 0.02 & 0.22 & 0.21 & 0.50 \\
\hline 3317.76 & 16.14 & 85.53 & 4.61 & 0.13 & 2.27 & 0.02 & 0.21 & 0.20 & 0.52 \\
\hline 3317.91 & 16.46 & 84.41 & 4.66 & 0.13 & 2.28 & 0.03 & 0.20 & 0.20 & 0.53 \\
\hline 3318.06 & 16.99 & 83.29 & 4.72 & 0.13 & 2.30 & 0.03 & 0.20 & 0.19 & 0.55 \\
\hline 3318.22 & 17.71 & 81.99 & 4.70 & 0.13 & 2.32 & 0.03 & 0.19 & 0.18 & 0.57 \\
\hline 3318.37 & 18.13 & 80.76 & 4.56 & 0.13 & 2.33 & 0.03 & 0.19 & 0.18 & 0.60 \\
\hline 3318.52 & 17.69 & 79.69 & 4.30 & 0.14 & 2.34 & 0.03 & 0.18 & 0.18 & 0.63 \\
\hline 3318.67 & 16.72 & 78.84 & 3.99 & 0.15 & 2.35 & 0.03 & 0.19 & 0.18 & 0.65 \\
\hline 3318.82 & 16.12 & 78.14 & 3.76 & 0.16 & 2.36 & 0.02 & 0.19 & 0.18 & 0.66 \\
\hline 3318.98 & 16.63 & 77.71 & 3.64 & 0.16 & 2.37 & 0.03 & 0.18 & 0.18 & 0.68 \\
\hline 3319.13 & 18.10 & 77.77 & 3.65 & 0.16 & 2.38 & 0.03 & 0.18 & 0.17 & 0.71 \\
\hline 3319.28 & 19.95 & 78.09 & 3.71 & 0.15 & 2.39 & 0.04 & 0.17 & 0.16 & 0.74 \\
\hline 3319.43 & 21.27 & 78.40 & 3.72 & 0.15 & 2.39 & 0.05 & 0.17 & 0.16 & 0.75 \\
\hline 3319.58 & 21.23 & 78.75 & 3.66 & 0.15 & 2.39 & 0.05 & 0.17 & 0.16 & 0.75 \\
\hline 3319.74 & 20.14 & 79.31 & 3.65 & 0.15 & 2.39 & 0.04 & 0.17 & 0.16 & 0.75 \\
\hline 3319.89 & 19.31 & 79.59 & 3.69 & 0.14 & 2.39 & 0.04 & 0.17 & 0.16 & 0.75 \\
\hline 3320.04 & 19.94 & 79.16 & 3.76 & 0.14 & 2.39 & 0.04 & 0.17 & 0.16 & 0.75 \\
\hline 3320.19 & 21.52 & 78.61 & 3.87 & 0.14 & 2.39 & 0.05 & 0.17 & 0.16 & 0.74 \\
\hline 3320.34 & 22.63 & 78.54 & 4.16 & 0.15 & 2.39 & 0.05 & 0.17 & 0.16 & 0.70 \\
\hline 3320.50 & 22.13 & 78.36 & 4.69 & 0.16 & 2.39 & 0.05 & 0.18 & 0.16 & 0.65 \\
\hline 3320.65 & 20.13 & 77.48 & 5.37 & 0.15 & 2.39 & 0.04 & 0.17 & 0.16 & 0.61 \\
\hline 3320.80 & 18.19 & 76.55 & 6.04 & 0.14 & 2.39 & 0.03 & 0.17 & 0.16 & 0.58 \\
\hline 3320.95 & 17.57 & 76.20 & 6.57 & 0.12 & 2.38 & 0.03 & 0.17 & 0.16 & 0.57 \\
\hline 3321.10 & 18.32 & 76.39 & 6.91 & 0.12 & 2.37 & 0.03 & 0.16 & 0.15 & 0.56 \\
\hline 3321.26 & 19.02 & 77.06 & 7.28 & 0.12 & 2.38 & 0.04 & 0.16 & 0.15 & 0.56 \\
\hline 3321.41 & 18.66 & 77.51 & 7.78 & 0.12 & 2.40 & 0.03 & 0.16 & 0.15 & 0.56 \\
\hline 3321.56 & 17.72 & 77.06 & 7.97 & 0.11 & 2.42 & 0.03 & 0.15 & 0.14 & 0.59 \\
\hline 3321.71 & 17.65 & 76.33 & 7.51 & 0.11 & 2.43 & 0.03 & 0.14 & 0.13 & 0.63 \\
\hline 3321.86 & 19.22 & 75.87 & 6.77 & 0.11 & 2.43 & 0.04 & 0.14 & 0.13 & 0.66 \\
\hline 3322.02 & 21.12 & 75.71 & 6.40 & 0.12 & 2.43 & 0.04 & 0.15 & 0.14 & 0.67 \\
\hline
\end{tabular}


Table 1 (continued)

\begin{tabular}{|c|c|c|c|c|c|c|c|c|c|}
\hline \multicolumn{6}{|c|}{ Input well log data } & \multicolumn{4}{|l|}{ Output results } \\
\hline Depth (ft) & $\begin{array}{l}\text { Gamma } \\
\text { ray }(\mathrm{API})\end{array}$ & Sonic (US/F) & $\begin{array}{l}\text { Deep } \\
\text { resistivity } \\
\left(\Omega \mathrm{m}^{2} / \mathrm{m}\right)\end{array}$ & $\begin{array}{l}\text { Neutron } \\
\text { porosity }\end{array}$ & Density (G/C3) & Shale volume & Total porosity & $\begin{array}{l}\text { Effective } \\
\text { porosity }\end{array}$ & Water saturation \\
\hline 3322.17 & 21.37 & 75.95 & 6.61 & 0.13 & 2.42 & 0.05 & 0.15 & 0.14 & 0.63 \\
\hline 3322.32 & 19.78 & 76.36 & 7.11 & 0.13 & 2.41 & 0.04 & 0.16 & 0.15 & 0.59 \\
\hline 3322.47 & 18.12 & 77.03 & 7.43 & 0.13 & 2.40 & 0.03 & 0.16 & 0.15 & 0.56 \\
\hline 3322.62 & 17.80 & 78.01 & 7.44 & 0.13 & 2.40 & 0.03 & 0.16 & 0.15 & 0.55 \\
\hline 3322.78 & 18.42 & 78.63 & 7.35 & 0.13 & 2.40 & 0.03 & 0.16 & 0.15 & 0.55 \\
\hline 3322.93 & 18.82 & 78.68 & 7.38 & 0.13 & 2.40 & 0.03 & 0.16 & 0.15 & 0.55 \\
\hline 3323.08 & 18.62 & 78.33 & 7.58 & 0.14 & 2.40 & 0.03 & 0.17 & 0.16 & 0.53 \\
\hline 3323.23 & 18.38 & 77.43 & 7.94 & 0.14 & 2.39 & 0.03 & 0.17 & 0.16 & 0.51 \\
\hline 3323.38 & 18.55 & 75.81 & 8.38 & 0.14 & 2.38 & 0.03 & 0.17 & 0.16 & 0.49 \\
\hline 3323.54 & 19.01 & 73.92 & 8.75 & 0.14 & 2.39 & 0.04 & 0.17 & 0.16 & 0.48 \\
\hline 3323.69 & 19.07 & 72.85 & 8.88 & 0.14 & 2.40 & 0.04 & 0.16 & 0.15 & 0.50 \\
\hline 3323.84 & 18.68 & 73.33 & 8.59 & 0.14 & 2.43 & 0.03 & 0.15 & 0.14 & 0.54 \\
\hline 3323.99 & 18.82 & 75.03 & 8.02 & 0.13 & 2.43 & 0.03 & 0.15 & 0.14 & 0.59 \\
\hline 3324.14 & 20.02 & 77.22 & 7.51 & 0.13 & 2.43 & 0.04 & 0.15 & 0.14 & 0.60 \\
\hline 3324.30 & 21.59 & 78.86 & 7.18 & 0.13 & 2.42 & 0.05 & 0.16 & 0.14 & 0.60 \\
\hline 3324.45 & 22.64 & 79.30 & 6.90 & 0.14 & 2.42 & 0.05 & 0.16 & 0.14 & 0.60 \\
\hline 3324.60 & 23.18 & 79.09 & 6.57 & 0.14 & 2.42 & 0.05 & 0.16 & 0.14 & 0.63 \\
\hline 3324.75 & 23.48 & 78.88 & 6.36 & 0.14 & 2.43 & 0.06 & 0.15 & 0.14 & 0.65 \\
\hline 3324.90 & 23.23 & 78.67 & 6.32 & 0.14 & 2.44 & 0.05 & 0.15 & 0.14 & 0.67 \\
\hline 3325.06 & 22.74 & 78.29 & 6.33 & 0.14 & 2.45 & 0.05 & 0.15 & 0.13 & 0.68 \\
\hline 3325.21 & 22.76 & 77.75 & 6.32 & 0.14 & 2.45 & 0.05 & 0.15 & 0.13 & 0.68 \\
\hline 3325.36 & 23.26 & 77.40 & 6.33 & 0.14 & 2.45 & 0.05 & 0.15 & 0.13 & 0.68 \\
\hline 3325.51 & 23.32 & 77.42 & 6.29 & 0.14 & 2.45 & 0.05 & 0.15 & 0.13 & 0.68 \\
\hline 3325.66 & 22.36 & 77.64 & 6.09 & 0.14 & 2.44 & 0.05 & 0.15 & 0.14 & 0.68 \\
\hline 3325.82 & 21.00 & 77.86 & 5.78 & 0.14 & 2.43 & 0.04 & 0.16 & 0.14 & 0.67 \\
\hline 3325.97 & 19.94 & 78.17 & 5.59 & 0.14 & 2.42 & 0.04 & 0.16 & 0.15 & 0.65 \\
\hline 3326.12 & 19.15 & 78.59 & 5.75 & 0.15 & 2.41 & 0.04 & 0.16 & 0.15 & 0.63 \\
\hline 3326.27 & 18.34 & 78.61 & 6.44 & 0.14 & 2.42 & 0.03 & 0.16 & 0.15 & 0.60 \\
\hline 3326.42 & 17.52 & 77.87 & 7.74 & 0.15 & 2.40 & 0.03 & 0.17 & 0.16 & 0.52 \\
\hline 3326.58 & 17.26 & 76.96 & 9.49 & 0.15 & 2.40 & 0.03 & 0.17 & 0.16 & 0.47 \\
\hline 3326.73 & 17.90 & 76.72 & 11.25 & 0.14 & 2.40 & 0.03 & 0.16 & 0.16 & 0.44 \\
\hline 3326.88 & 18.86 & 77.42 & 12.65 & 0.13 & 2.41 & 0.04 & 0.16 & 0.15 & 0.43 \\
\hline 3327.03 & 19.34 & 78.75 & 13.67 & 0.12 & 2.41 & 0.04 & 0.15 & 0.14 & 0.43 \\
\hline 3327.18 & 19.17 & 79.75 & 14.36 & 0.11 & 2.40 & 0.04 & 0.15 & 0.14 & 0.43 \\
\hline 3327.34 & 18.93 & 79.78 & 14.54 & 0.11 & 2.40 & 0.04 & 0.15 & 0.14 & 0.42 \\
\hline 3327.49 & 19.16 & 79.20 & 14.05 & 0.11 & 2.39 & 0.04 & 0.16 & 0.15 & 0.42 \\
\hline 3327.64 & 19.52 & 78.58 & 13.40 & 0.12 & 2.39 & 0.04 & 0.16 & 0.15 & 0.42 \\
\hline 3327.79 & 19.46 & 78.26 & 13.33 & 0.13 & 2.39 & 0.04 & 0.16 & 0.15 & 0.41 \\
\hline 3327.94 & 18.64 & 78.29 & 13.93 & 0.13 & 2.40 & 0.03 & 0.16 & 0.15 & 0.41 \\
\hline 3328.10 & 17.42 & 78.72 & 14.63 & 0.13 & 2.41 & 0.03 & 0.16 & 0.15 & 0.40 \\
\hline 3328.25 & 16.92 & 79.36 & 14.85 & 0.13 & 2.42 & 0.03 & 0.16 & 0.15 & 0.40 \\
\hline 3328.40 & 17.59 & 79.81 & 14.63 & 0.13 & 2.41 & 0.03 & 0.16 & 0.15 & 0.40 \\
\hline 3328.55 & 18.55 & 79.85 & 14.75 & 0.13 & 2.39 & 0.03 & 0.16 & 0.15 & 0.38 \\
\hline 3328.70 & 18.93 & 79.32 & 15.52 & 0.12 & 2.37 & 0.04 & 0.17 & 0.16 & 0.37 \\
\hline 3328.86 & 19.22 & 78.38 & 16.17 & 0.12 & 2.36 & 0.04 & 0.17 & 0.16 & 0.36 \\
\hline 3329.01 & 19.91 & 77.43 & 15.75 & 0.12 & 2.38 & 0.04 & 0.16 & 0.15 & 0.37 \\
\hline 3329.16 & 20.42 & 76.66 & 14.43 & 0.12 & 2.40 & 0.04 & 0.16 & 0.15 & 0.40 \\
\hline
\end{tabular}


Table 1 (continued)

\begin{tabular}{|c|c|c|c|c|c|c|c|c|c|}
\hline \multicolumn{6}{|c|}{ Input well log data } & \multicolumn{4}{|l|}{ Output results } \\
\hline Depth (ft) & $\begin{array}{l}\text { Gamma } \\
\text { ray (API) }\end{array}$ & Sonic (US/F) & $\begin{array}{l}\text { Deep } \\
\text { resistivity } \\
\left(\Omega \mathrm{m}^{2} / \mathrm{m}\right)\end{array}$ & $\begin{array}{l}\text { Neutron } \\
\text { porosity }\end{array}$ & Density (G/C3) & Shale volume & Total porosity & $\begin{array}{l}\text { Effective } \\
\text { porosity }\end{array}$ & Water saturation \\
\hline 3329.31 & 20.03 & 76.21 & 13.88 & 0.13 & 2.41 & 0.04 & 0.16 & 0.15 & 0.41 \\
\hline 3329.46 & 18.91 & 76.44 & 15.52 & 0.14 & 2.41 & 0.04 & 0.16 & 0.15 & 0.39 \\
\hline 3329.62 & 17.83 & 77.42 & 18.85 & 0.14 & 2.41 & 0.03 & 0.16 & 0.15 & 0.34 \\
\hline 3329.77 & 17.18 & 78.75 & 21.94 & 0.14 & 2.40 & 0.03 & 0.16 & 0.15 & 0.31 \\
\hline 3329.92 & 16.76 & 79.92 & 23.48 & 0.13 & 2.40 & 0.03 & 0.16 & 0.15 & 0.30 \\
\hline 3330.07 & 16.10 & 80.42 & 23.56 & 0.12 & 2.39 & 0.02 & 0.16 & 0.16 & 0.30 \\
\hline 3330.22 & 15.25 & 80.23 & 22.28 & 0.11 & 2.37 & 0.02 & 0.16 & 0.16 & 0.31 \\
\hline 3330.38 & 14.97 & 79.76 & 19.56 & 0.11 & 2.36 & 0.02 & 0.17 & 0.16 & 0.32 \\
\hline 3330.53 & 15.53 & 79.38 & 16.39 & 0.11 & 2.35 & 0.02 & 0.17 & 0.16 & 0.35 \\
\hline 3330.68 & 16.44 & 79.29 & 14.33 & 0.11 & 2.36 & 0.03 & 0.17 & 0.16 & 0.38 \\
\hline 3330.83 & 17.12 & 79.49 & 13.53 & 0.11 & 2.37 & 0.03 & 0.17 & 0.16 & 0.40 \\
\hline 3330.98 & 17.50 & 79.74 & 13.12 & 0.11 & 2.38 & 0.03 & 0.16 & 0.15 & 0.41 \\
\hline 3331.14 & 18.07 & 79.79 & 12.65 & 0.12 & 2.38 & 0.03 & 0.16 & 0.15 & 0.42 \\
\hline 3331.29 & 18.99 & 79.62 & 12.16 & 0.12 & 2.38 & 0.04 & 0.16 & 0.15 & 0.43 \\
\hline 3331.44 & 20.10 & 79.38 & 11.81 & 0.12 & 2.38 & 0.04 & 0.16 & 0.15 & 0.43 \\
\hline 3331.59 & 20.99 & 79.15 & 11.65 & 0.13 & 2.38 & 0.04 & 0.17 & 0.15 & 0.44 \\
\hline 3331.74 & 20.98 & 78.90 & 11.51 & 0.13 & 2.38 & 0.04 & 0.17 & 0.15 & 0.44 \\
\hline 3331.90 & 19.96 & 78.71 & 11.14 & 0.13 & 2.39 & 0.04 & 0.17 & 0.15 & 0.44 \\
\hline 3332.05 & 18.77 & 78.61 & 10.54 & 0.13 & 2.39 & 0.03 & 0.17 & 0.16 & 0.45 \\
\hline 3332.20 & 18.29 & 78.37 & 9.89 & 0.13 & 2.39 & 0.03 & 0.17 & 0.16 & 0.46 \\
\hline 3332.35 & 18.54 & 77.69 & 9.33 & 0.13 & 2.39 & 0.03 & 0.17 & 0.16 & 0.48 \\
\hline 3332.50 & 18.82 & 76.53 & 8.81 & 0.13 & 2.40 & 0.03 & 0.16 & 0.15 & 0.51 \\
\hline 3332.66 & 19.08 & 75.65 & 7.94 & 0.13 & 2.40 & 0.04 & 0.16 & 0.15 & 0.54 \\
\hline 3332.81 & 19.69 & 75.73 & 6.67 & 0.13 & 2.41 & 0.04 & 0.16 & 0.15 & 0.60 \\
\hline 3332.96 & 20.39 & 76.51 & 5.53 & 0.13 & 2.42 & 0.04 & 0.15 & 0.14 & 0.69 \\
\hline 3333.11 & 21.23 & 77.54 & 4.98 & 0.12 & 2.43 & 0.05 & 0.15 & 0.13 & 0.77 \\
\hline 3333.26 & 22.18 & 78.15 & 4.89 & 0.12 & 2.42 & 0.05 & 0.15 & 0.13 & 0.79 \\
\hline 3333.42 & 22.71 & 77.88 & 4.95 & 0.12 & 2.41 & 0.05 & 0.15 & 0.14 & 0.75 \\
\hline 3333.57 & 22.12 & 77.09 & 5.06 & 0.13 & 2.41 & 0.05 & 0.16 & 0.14 & 0.70 \\
\hline 3333.72 & 20.83 & 76.27 & 5.38 & 0.14 & 2.42 & 0.04 & 0.16 & 0.15 & 0.68 \\
\hline 3333.87 & 20.13 & 75.56 & 6.19 & 0.14 & 2.43 & 0.04 & 0.16 & 0.14 & 0.64 \\
\hline 3334.02 & 19.81 & 75.13 & 7.99 & 0.14 & 2.43 & 0.04 & 0.16 & 0.14 & 0.56 \\
\hline 3334.18 & 19.04 & 75.18 & 11.23 & 0.14 & 2.43 & 0.04 & 0.16 & 0.14 & 0.47 \\
\hline 3334.33 & 18.24 & 75.70 & 15.72 & 0.14 & 2.43 & 0.03 & 0.15 & 0.14 & 0.40 \\
\hline 3334.48 & 18.46 & 76.44 & 20.96 & 0.13 & 2.44 & 0.03 & 0.15 & 0.14 & 0.37 \\
\hline 3334.63 & 19.56 & 77.19 & 27.66 & 0.12 & 2.45 & 0.04 & 0.14 & 0.13 & 0.34 \\
\hline 3334.78 & 20.00 & 77.75 & 36.89 & 0.10 & 2.44 & 0.04 & 0.13 & 0.12 & 0.30 \\
\hline 3334.94 & 19.64 & 77.96 & 48.26 & 0.09 & 2.42 & 0.04 & 0.14 & 0.13 & 0.25 \\
\hline 3335.09 & 19.52 & 77.99 & 61.76 & 0.09 & 2.38 & 0.04 & 0.15 & 0.14 & 0.20 \\
\hline 3335.24 & 19.73 & 78.12 & 74.65 & 0.09 & 2.37 & 0.04 & 0.16 & 0.14 & 0.18 \\
\hline 3335.39 & 19.48 & 78.39 & 76.10 & 0.09 & 2.37 & 0.04 & 0.15 & 0.14 & 0.18 \\
\hline 3335.54 & 18.39 & 78.77 & 62.92 & 0.09 & 2.38 & 0.03 & 0.15 & 0.14 & 0.20 \\
\hline 3335.70 & 17.33 & 79.23 & 47.17 & 0.09 & 2.38 & 0.03 & 0.15 & 0.14 & 0.23 \\
\hline 3335.85 & 17.01 & 79.43 & 36.15 & 0.09 & 2.38 & 0.03 & 0.15 & 0.15 & 0.26 \\
\hline 3336.00 & 16.89 & 79.19 & 28.76 & 0.10 & 2.37 & 0.03 & 0.16 & 0.15 & 0.28 \\
\hline 3336.15 & 16.37 & 78.55 & 24.69 & 0.10 & 2.37 & 0.02 & 0.16 & 0.15 & 0.30 \\
\hline 3336.30 & 16.09 & 77.56 & 22.64 & 0.10 & 2.37 & 0.02 & 0.16 & 0.15 & 0.32 \\
\hline
\end{tabular}


Table 1 (continued)

\begin{tabular}{|c|c|c|c|c|c|c|c|c|c|}
\hline \multicolumn{6}{|c|}{ Input well log data } & \multicolumn{4}{|l|}{ Output results } \\
\hline Depth (ft) & $\begin{array}{l}\text { Gamma } \\
\text { ray }(\mathrm{API})\end{array}$ & Sonic (US/F) & $\begin{array}{l}\text { Deep } \\
\text { resistivity } \\
\left(\Omega \mathrm{m}^{2} / \mathrm{m}\right)\end{array}$ & $\begin{array}{l}\text { Neutron } \\
\text { porosity }\end{array}$ & Density (G/C3) & Shale volume & Total porosity & $\begin{array}{l}\text { Effective } \\
\text { porosity }\end{array}$ & Water saturation \\
\hline 3336.46 & 16.74 & 76.37 & 20.39 & 0.11 & 2.39 & 0.03 & 0.15 & 0.14 & 0.35 \\
\hline 3336.61 & 17.58 & 75.51 & 17.61 & 0.11 & 2.41 & 0.03 & 0.15 & 0.14 & 0.40 \\
\hline 3336.76 & 17.56 & 75.74 & 14.98 & 0.11 & 2.42 & 0.03 & 0.14 & 0.13 & 0.44 \\
\hline 3336.91 & 17.56 & 77.02 & 12.76 & 0.11 & 2.42 & 0.03 & 0.15 & 0.14 & 0.47 \\
\hline 3337.06 & 19.24 & 78.10 & 11.00 & 0.12 & 2.41 & 0.04 & 0.15 & 0.14 & 0.49 \\
\hline 3337.22 & 21.59 & 78.07 & 9.87 & 0.13 & 2.41 & 0.05 & 0.16 & 0.14 & 0.50 \\
\hline 3337.37 & 22.23 & 77.32 & 9.30 & 0.13 & 2.42 & 0.05 & 0.16 & 0.14 & 0.53 \\
\hline 3337.52 & 21.24 & 76.70 & 8.71 & 0.14 & 2.44 & 0.05 & 0.15 & 0.14 & 0.56 \\
\hline 3337.67 & 20.72 & 76.67 & 7.91 & 0.14 & 2.44 & 0.04 & 0.15 & 0.14 & 0.60 \\
\hline 3337.82 & 21.43 & 77.12 & 7.36 & 0.14 & 2.44 & 0.05 & 0.15 & 0.14 & 0.62 \\
\hline 3337.98 & 22.31 & 77.58 & 7.33 & 0.14 & 2.44 & 0.05 & 0.15 & 0.14 & 0.61 \\
\hline 3338.13 & 23.00 & 77.78 & 7.60 & 0.15 & 2.44 & 0.05 & 0.15 & 0.14 & 0.60 \\
\hline 3338.28 & 23.65 & 78.03 & 7.91 & 0.15 & 2.45 & 0.06 & 0.15 & 0.13 & 0.60 \\
\hline 3338.43 & 23.33 & 78.40 & 8.17 & 0.15 & 2.45 & 0.05 & 0.15 & 0.13 & 0.59 \\
\hline 3338.58 & 21.52 & 78.66 & 8.38 & 0.15 & 2.45 & 0.05 & 0.15 & 0.14 & 0.56 \\
\hline 3338.74 & 19.48 & 78.36 & 8.60 & 0.15 & 2.44 & 0.04 & 0.16 & 0.15 & 0.54 \\
\hline 3338.89 & 18.74 & 77.42 & 8.96 & 0.15 & 2.44 & 0.03 & 0.16 & 0.15 & 0.52 \\
\hline 3339.04 & 19.15 & 76.45 & 9.47 & 0.15 & 2.44 & 0.04 & 0.16 & 0.14 & 0.51 \\
\hline 3339.19 & 19.45 & 75.59 & 9.82 & 0.14 & 2.44 & 0.04 & 0.15 & 0.14 & 0.52 \\
\hline 3339.34 & 19.52 & 74.74 & 9.77 & 0.14 & 2.45 & 0.04 & 0.15 & 0.14 & 0.54 \\
\hline 3339.50 & 19.96 & 74.24 & 9.50 & 0.14 & 2.46 & 0.04 & 0.14 & 0.13 & 0.56 \\
\hline 3339.65 & 20.71 & 74.09 & 9.22 & 0.14 & 2.46 & 0.04 & 0.14 & 0.13 & 0.59 \\
\hline 3339.80 & 21.32 & 74.06 & 8.88 & 0.14 & 2.47 & 0.05 & 0.14 & 0.13 & 0.61 \\
\hline 3339.95 & 21.40 & 74.28 & 8.62 & 0.13 & 2.47 & 0.05 & 0.14 & 0.12 & 0.63 \\
\hline 3340.10 & 21.07 & 74.72 & 8.76 & 0.13 & 2.47 & 0.04 & 0.14 & 0.12 & 0.63 \\
\hline 3340.26 & 20.55 & 75.08 & 9.42 & 0.13 & 2.47 & 0.04 & 0.14 & 0.12 & 0.60 \\
\hline 3340.41 & 19.76 & 75.41 & 10.37 & 0.13 & 2.46 & 0.04 & 0.14 & 0.13 & 0.55 \\
\hline 3340.56 & 18.81 & 76.20 & 11.21 & 0.13 & 2.43 & 0.03 & 0.15 & 0.14 & 0.49 \\
\hline 3340.71 & 18.02 & 77.51 & 11.65 & 0.13 & 2.39 & 0.03 & 0.16 & 0.15 & 0.43 \\
\hline 3340.86 & 17.78 & 79.00 & 11.73 & 0.12 & 2.34 & 0.03 & 0.18 & 0.17 & 0.39 \\
\hline 3341.02 & 17.96 & 80.47 & 11.72 & 0.11 & 2.31 & 0.03 & 0.19 & 0.18 & 0.37 \\
\hline 3341.17 & 17.86 & 81.64 & 11.69 & 0.12 & 2.29 & 0.03 & 0.20 & 0.19 & 0.36 \\
\hline 3341.32 & 17.34 & 82.18 & 11.49 & 0.12 & 2.29 & 0.03 & 0.20 & 0.19 & 0.35 \\
\hline 3341.47 & 17.03 & 81.79 & 11.10 & 0.12 & 2.28 & 0.03 & 0.20 & 0.19 & 0.35 \\
\hline 3341.62 & 17.18 & 80.32 & 10.56 & 0.12 & 2.28 & 0.03 & 0.20 & 0.19 & 0.36 \\
\hline 3341.78 & 17.60 & 78.48 & 9.92 & 0.12 & 2.29 & 0.03 & 0.20 & 0.19 & 0.38 \\
\hline 3341.93 & 18.32 & 77.12 & 9.50 & 0.12 & 2.31 & 0.03 & 0.19 & 0.18 & 0.41 \\
\hline 3342.08 & 19.41 & 76.22 & 9.63 & 0.11 & 2.34 & 0.04 & 0.17 & 0.16 & 0.45 \\
\hline 3342.23 & 19.95 & 75.99 & 10.04 & 0.11 & 2.37 & 0.04 & 0.16 & 0.15 & 0.48 \\
\hline 3342.38 & 18.64 & 76.75 & 10.27 & 0.11 & 2.39 & 0.03 & 0.16 & 0.15 & 0.49 \\
\hline 3342.54 & 16.17 & 77.82 & 10.03 & 0.11 & 2.40 & 0.02 & 0.15 & 0.15 & 0.50 \\
\hline 3342.69 & 15.02 & 78.65 & 9.29 & 0.11 & 2.40 & 0.02 & 0.15 & 0.15 & 0.52 \\
\hline 3342.84 & 16.92 & 79.34 & 8.51 & 0.11 & 2.39 & 0.03 & 0.15 & 0.15 & 0.54 \\
\hline 3342.99 & 20.03 & 79.85 & 7.91 & 0.11 & 2.38 & 0.04 & 0.16 & 0.15 & 0.54 \\
\hline 3343.14 & 20.89 & 80.25 & 7.42 & 0.12 & 2.37 & 0.04 & 0.17 & 0.15 & 0.54 \\
\hline 3343.30 & 19.15 & 80.64 & 7.16 & 0.13 & 2.37 & 0.04 & 0.17 & 0.16 & 0.54 \\
\hline 3343.45 & 17.40 & 80.85 & 7.11 & 0.13 & 2.38 & 0.03 & 0.17 & 0.16 & 0.54 \\
\hline
\end{tabular}


Table 1 (continued)

\begin{tabular}{|c|c|c|c|c|c|c|c|c|c|}
\hline \multicolumn{6}{|c|}{ Input well log data } & \multicolumn{4}{|l|}{ Output results } \\
\hline Depth (ft) & $\begin{array}{l}\text { Gamma } \\
\text { ray }(A P I)\end{array}$ & Sonic (US/F) & $\begin{array}{l}\text { Deep } \\
\text { resistivity } \\
\left(\Omega \mathrm{m}^{2} / \mathrm{m}\right)\end{array}$ & $\begin{array}{l}\text { Neutron } \\
\text { porosity }\end{array}$ & Density (G/C3) & Shale volume & Total porosity & $\begin{array}{l}\text { Effective } \\
\text { porosity }\end{array}$ & Water saturation \\
\hline 3343.60 & 17.67 & 80.89 & 7.07 & 0.14 & 2.39 & 0.03 & 0.17 & 0.16 & 0.55 \\
\hline 3343.75 & 19.03 & 80.92 & 6.90 & 0.14 & 2.41 & 0.04 & 0.16 & 0.15 & 0.57 \\
\hline 3343.90 & 19.72 & 80.86 & 6.68 & 0.15 & 2.42 & 0.04 & 0.16 & 0.15 & 0.60 \\
\hline 3344.06 & 19.70 & 80.96 & 6.49 & 0.15 & 2.42 & 0.04 & 0.16 & 0.15 & 0.60 \\
\hline 3344.21 & 19.28 & 81.59 & 6.35 & 0.15 & 2.40 & 0.04 & 0.17 & 0.16 & 0.58 \\
\hline 3344.36 & 18.37 & 82.24 & 6.21 & 0.15 & 2.37 & 0.03 & 0.18 & 0.17 & 0.55 \\
\hline 3344.51 & 17.10 & 82.36 & 6.05 & 0.14 & 2.34 & 0.03 & 0.19 & 0.18 & 0.52 \\
\hline 3344.66 & 16.44 & 82.13 & 6.09 & 0.14 & 2.32 & 0.03 & 0.20 & 0.19 & 0.49 \\
\hline 3344.82 & 17.10 & 81.86 & 6.45 & 0.14 & 2.31 & 0.03 & 0.20 & 0.19 & 0.47 \\
\hline 3344.97 & 18.01 & 81.88 & 6.88 & 0.14 & 2.31 & 0.03 & 0.20 & 0.19 & 0.46 \\
\hline 3345.12 & 17.90 & 82.44 & 7.01 & 0.13 & 2.31 & 0.03 & 0.20 & 0.19 & 0.46 \\
\hline 3345.27 & 17.18 & 83.16 & 6.78 & 0.13 & 2.30 & 0.03 & 0.20 & 0.19 & 0.46 \\
\hline 3345.42 & 17.41 & 83.33 & 6.42 & 0.13 & 2.29 & 0.03 & 0.20 & 0.19 & 0.47 \\
\hline 3345.58 & 18.80 & 83.00 & 6.11 & 0.13 & 2.30 & 0.03 & 0.20 & 0.19 & 0.49 \\
\hline 3345.73 & 19.84 & 82.70 & 5.80 & 0.13 & 2.32 & 0.04 & 0.19 & 0.18 & 0.53 \\
\hline 3345.88 & 19.47 & 82.69 & 5.39 & 0.14 & 2.34 & 0.04 & 0.19 & 0.18 & 0.56 \\
\hline 3346.03 & 18.67 & 82.97 & 4.97 & 0.15 & 2.35 & 0.03 & 0.19 & 0.18 & 0.58 \\
\hline 3346.18 & 18.95 & 83.43 & 4.70 & 0.15 & 2.35 & 0.04 & 0.19 & 0.18 & 0.58 \\
\hline 3346.34 & 19.74 & 83.82 & 4.70 & 0.16 & 2.35 & 0.04 & 0.19 & 0.18 & 0.58 \\
\hline 3346.49 & 19.59 & 83.98 & 5.05 & 0.16 & 2.34 & 0.04 & 0.20 & 0.18 & 0.55 \\
\hline 3346.64 & 18.07 & 84.21 & 5.80 & 0.16 & 2.33 & 0.03 & 0.20 & 0.19 & 0.49 \\
\hline 3346.79 & 16.27 & 84.61 & 6.73 & 0.15 & 2.30 & 0.02 & 0.21 & 0.20 & 0.44 \\
\hline 3346.94 & 15.51 & 85.03 & 7.46 & 0.14 & 2.28 & 0.02 & 0.21 & 0.20 & 0.41 \\
\hline 3347.10 & 15.60 & 85.29 & 7.88 & 0.13 & 2.27 & 0.02 & 0.21 & 0.20 & 0.40 \\
\hline 3347.25 & 15.56 & 84.83 & 8.00 & 0.12 & 2.27 & 0.02 & 0.21 & 0.20 & 0.40 \\
\hline 3347.40 & 14.97 & 83.69 & 7.80 & 0.12 & 2.28 & 0.02 & 0.20 & 0.20 & 0.42 \\
\hline 3347.55 & 14.30 & 82.73 & 7.59 & 0.13 & 2.30 & 0.02 & 0.20 & 0.19 & 0.43 \\
\hline 3347.70 & 13.90 & 82.20 & 7.74 & 0.13 & 2.31 & 0.01 & 0.20 & 0.19 & 0.43 \\
\hline 3347.86 & 13.59 & 82.00 & 8.10 & 0.14 & 2.31 & 0.01 & 0.20 & 0.19 & 0.42 \\
\hline 3348.01 & 13.62 & 82.17 & 8.14 & 0.14 & 2.32 & 0.01 & 0.20 & 0.19 & 0.42 \\
\hline 3348.16 & 14.33 & 82.34 & 7.66 & 0.15 & 2.33 & 0.02 & 0.19 & 0.19 & 0.44 \\
\hline 3348.31 & 15.19 & 82.23 & 6.97 & 0.14 & 2.34 & 0.02 & 0.19 & 0.18 & 0.48 \\
\hline 3348.46 & 15.57 & 82.18 & 6.39 & 0.13 & 2.34 & 0.02 & 0.18 & 0.18 & 0.51 \\
\hline 3348.62 & 16.00 & 82.08 & 6.07 & 0.13 & 2.34 & 0.02 & 0.18 & 0.17 & 0.54 \\
\hline 3348.77 & 16.88 & 81.62 & 6.04 & 0.13 & 2.35 & 0.03 & 0.18 & 0.17 & 0.54 \\
\hline 3348.92 & 17.29 & 81.05 & 6.23 & 0.13 & 2.35 & 0.03 & 0.18 & 0.17 & 0.53 \\
\hline 3349.07 & 16.51 & 80.40 & 6.45 & 0.14 & 2.35 & 0.03 & 0.18 & 0.17 & 0.52 \\
\hline 3349.22 & 15.57 & 79.64 & 6.58 & 0.13 & 2.35 & 0.02 & 0.18 & 0.17 & 0.51 \\
\hline 3349.38 & 15.90 & 79.17 & 6.61 & 0.13 & 2.35 & 0.02 & 0.18 & 0.17 & 0.51 \\
\hline 3349.53 & 17.38 & 78.90 & 6.62 & 0.14 & 2.36 & 0.03 & 0.18 & 0.17 & 0.52 \\
\hline 3349.68 & 19.12 & 78.61 & 6.63 & 0.14 & 2.37 & 0.04 & 0.18 & 0.17 & 0.53 \\
\hline 3349.83 & 20.53 & 78.44 & 6.70 & 0.15 & 2.39 & 0.04 & 0.17 & 0.16 & 0.55 \\
\hline 3349.98 & 21.38 & 78.19 & 6.88 & 0.15 & 2.40 & 0.05 & 0.17 & 0.15 & 0.56 \\
\hline 3350.14 & 21.35 & 77.90 & 7.20 & 0.15 & 2.41 & 0.05 & 0.16 & 0.15 & 0.56 \\
\hline 3350.29 & 20.00 & 77.96 & 7.62 & 0.15 & 2.42 & 0.04 & 0.16 & 0.15 & 0.55 \\
\hline 3350.44 & 17.73 & 78.28 & 8.26 & 0.15 & 2.42 & 0.03 & 0.16 & 0.15 & 0.52 \\
\hline 3350.59 & 15.74 & 78.58 & 9.20 & 0.14 & 2.40 & 0.02 & 0.17 & 0.16 & 0.48 \\
\hline
\end{tabular}


Table 1 (continued)

\begin{tabular}{|c|c|c|c|c|c|c|c|c|c|}
\hline \multicolumn{6}{|c|}{ Input well log data } & \multicolumn{4}{|l|}{ Output results } \\
\hline Depth (ft) & $\begin{array}{l}\text { Gamma } \\
\text { ray }(\mathrm{API})\end{array}$ & Sonic (US/F) & $\begin{array}{l}\text { Deep } \\
\text { resistivity } \\
\left(\Omega \mathrm{m}^{2} / \mathrm{m}\right)\end{array}$ & $\begin{array}{l}\text { Neutron } \\
\text { porosity }\end{array}$ & Density (G/C3) & Shale volume & Total porosity & $\begin{array}{l}\text { Effective } \\
\text { porosity }\end{array}$ & Water saturation \\
\hline 3350.74 & 15.09 & 78.69 & 10.29 & 0.13 & 2.39 & 0.02 & 0.17 & 0.16 & 0.45 \\
\hline 3350.90 & 15.74 & 78.51 & 11.26 & 0.12 & 2.38 & 0.02 & 0.17 & 0.16 & 0.43 \\
\hline 3351.05 & 16.57 & 78.15 & 11.93 & 0.12 & 2.38 & 0.03 & 0.16 & 0.16 & 0.43 \\
\hline 3351.20 & 16.76 & 77.85 & 12.23 & 0.12 & 2.38 & 0.03 & 0.16 & 0.15 & 0.43 \\
\hline 3351.35 & 16.47 & 77.60 & 12.18 & 0.12 & 2.38 & 0.03 & 0.16 & 0.15 & 0.43 \\
\hline 3351.50 & 16.13 & 77.32 & 11.95 & 0.12 & 2.38 & 0.02 & 0.16 & 0.15 & 0.43 \\
\hline 3351.66 & 16.14 & 77.11 & 11.71 & 0.12 & 2.38 & 0.02 & 0.16 & 0.15 & 0.44 \\
\hline 3351.81 & 16.45 & 76.90 & 11.34 & 0.12 & 2.39 & 0.03 & 0.16 & 0.15 & 0.45 \\
\hline 3351.96 & 16.52 & 76.64 & 10.76 & 0.12 & 2.40 & 0.03 & 0.16 & 0.15 & 0.47 \\
\hline 3352.11 & 16.20 & 76.38 & 10.06 & 0.12 & 2.40 & 0.02 & 0.16 & 0.15 & 0.49 \\
\hline 3352.26 & 16.08 & 76.26 & 9.35 & 0.12 & 2.40 & 0.02 & 0.16 & 0.15 & 0.51 \\
\hline 3352.42 & 16.56 & 76.23 & 8.76 & 0.13 & 2.41 & 0.03 & 0.16 & 0.15 & 0.52 \\
\hline 3352.57 & 17.41 & 76.05 & 8.42 & 0.13 & 2.42 & 0.03 & 0.16 & 0.15 & 0.54 \\
\hline 3352.72 & 18.15 & 75.80 & 8.38 & 0.13 & 2.42 & 0.03 & 0.15 & 0.14 & 0.55 \\
\hline 3352.87 & 18.87 & 75.66 & 8.54 & 0.14 & 2.43 & 0.04 & 0.15 & 0.14 & 0.55 \\
\hline 3353.02 & 19.50 & 75.77 & 8.70 & 0.14 & 2.43 & 0.04 & 0.15 & 0.14 & 0.55 \\
\hline 3353.18 & 19.28 & 76.26 & 8.74 & 0.14 & 2.43 & 0.04 & 0.15 & 0.14 & 0.55 \\
\hline 3353.33 & 17.97 & 76.66 & 8.65 & 0.14 & 2.43 & 0.03 & 0.16 & 0.15 & 0.53 \\
\hline 3353.48 & 16.34 & 76.48 & 8.59 & 0.14 & 2.41 & 0.02 & 0.16 & 0.16 & 0.50 \\
\hline 3353.63 & 15.51 & 75.93 & 8.74 & 0.14 & 2.39 & 0.02 & 0.17 & 0.16 & 0.48 \\
\hline 3353.78 & 15.96 & 75.49 & 9.19 & 0.13 & 2.39 & 0.02 & 0.17 & 0.16 & 0.48 \\
\hline 3353.94 & 16.86 & 75.38 & 9.65 & 0.12 & 2.40 & 0.03 & 0.16 & 0.15 & 0.50 \\
\hline 3354.09 & 17.37 & 75.69 & 9.83 & 0.11 & 2.40 & 0.03 & 0.15 & 0.14 & 0.51 \\
\hline 3354.24 & 17.53 & 76.06 & 9.80 & 0.11 & 2.39 & 0.03 & 0.16 & 0.15 & 0.50 \\
\hline 3354.39 & 17.67 & 75.99 & 9.72 & 0.11 & 2.38 & 0.03 & 0.16 & 0.15 & 0.49 \\
\hline 3354.54 & 17.82 & 75.67 & 9.59 & 0.10 & 2.37 & 0.03 & 0.16 & 0.15 & 0.49 \\
\hline 3354.70 & 17.46 & 75.46 & 9.44 & 0.10 & 2.38 & 0.03 & 0.16 & 0.15 & 0.50 \\
\hline 3354.85 & 16.95 & 75.45 & 9.24 & 0.10 & 2.38 & 0.03 & 0.16 & 0.15 & 0.51 \\
\hline 3355.00 & 17.23 & 75.62 & 8.91 & 0.10 & 2.38 & 0.03 & 0.16 & 0.15 & 0.52 \\
\hline 3355.15 & 18.24 & 75.78 & 8.39 & 0.10 & 2.38 & 0.03 & 0.16 & 0.15 & 0.54 \\
\hline 3355.30 & 18.86 & 75.74 & 7.69 & 0.10 & 2.38 & 0.04 & 0.16 & 0.14 & 0.57 \\
\hline 3355.46 & 18.44 & 75.75 & 6.84 & 0.10 & 2.39 & 0.03 & 0.15 & 0.14 & 0.61 \\
\hline 3355.61 & 17.62 & 76.00 & 6.07 & 0.11 & 2.39 & 0.03 & 0.15 & 0.15 & 0.64 \\
\hline 3355.76 & 17.29 & 76.32 & 5.57 & 0.12 & 2.40 & 0.03 & 0.16 & 0.15 & 0.67 \\
\hline 3355.91 & 17.97 & 76.51 & 5.32 & 0.13 & 2.40 & 0.03 & 0.16 & 0.15 & 0.67 \\
\hline 3356.06 & 19.20 & 76.55 & 5.16 & 0.14 & 2.41 & 0.04 & 0.16 & 0.15 & 0.68 \\
\hline 3356.22 & 20.01 & 76.72 & 5.02 & 0.14 & 2.41 & 0.04 & 0.16 & 0.15 & 0.69 \\
\hline 3356.37 & 19.66 & 77.06 & 4.94 & 0.14 & 2.42 & 0.04 & 0.16 & 0.15 & 0.70 \\
\hline 3356.52 & 18.67 & 77.38 & 5.01 & 0.14 & 2.41 & 0.03 & 0.16 & 0.15 & 0.69 \\
\hline 3356.67 & 18.30 & 77.62 & 5.24 & 0.13 & 2.41 & 0.03 & 0.16 & 0.15 & 0.67 \\
\hline 3356.82 & 18.52 & 77.70 & 5.47 & 0.13 & 2.40 & 0.03 & 0.16 & 0.15 & 0.65 \\
\hline 3356.98 & 18.71 & 77.71 & 5.68 & 0.14 & 2.40 & 0.03 & 0.16 & 0.15 & 0.63 \\
\hline 3357.13 & 18.58 & 77.77 & 5.95 & 0.14 & 2.40 & 0.03 & 0.16 & 0.15 & 0.61 \\
\hline 3357.28 & 18.10 & 77.80 & 6.22 & 0.15 & 2.41 & 0.03 & 0.17 & 0.16 & 0.59 \\
\hline 3357.43 & 17.31 & 77.70 & 6.34 & 0.15 & 2.40 & 0.03 & 0.17 & 0.16 & 0.57 \\
\hline 3357.58 & 16.68 & 77.43 & 6.28 & 0.16 & 2.39 & 0.03 & 0.17 & 0.17 & 0.55 \\
\hline 3357.74 & 16.82 & 77.17 & 6.08 & 0.15 & 2.38 & 0.03 & 0.18 & 0.17 & 0.54 \\
\hline
\end{tabular}


Table 1 (continued)

\begin{tabular}{|c|c|c|c|c|c|c|c|c|c|}
\hline \multicolumn{6}{|c|}{ Input well log data } & \multicolumn{4}{|l|}{ Output results } \\
\hline Depth (ft) & $\begin{array}{l}\text { Gamma } \\
\text { ray }(\mathrm{API})\end{array}$ & Sonic (US/F) & $\begin{array}{l}\text { Deep } \\
\text { resistivity } \\
\left(\Omega \mathrm{m}^{2} / \mathrm{m}\right)\end{array}$ & $\begin{array}{l}\text { Neutron } \\
\text { porosity }\end{array}$ & Density (G/C3) & Shale volume & Total porosity & $\begin{array}{l}\text { Effective } \\
\text { porosity }\end{array}$ & Water saturation \\
\hline 3357.89 & 17.33 & 77.08 & 5.71 & 0.15 & 2.37 & 0.03 & 0.18 & 0.17 & 0.56 \\
\hline 3358.04 & 17.55 & 77.21 & 5.16 & 0.14 & 2.38 & 0.03 & 0.17 & 0.17 & 0.61 \\
\hline 3358.19 & 17.26 & 77.75 & 4.54 & 0.14 & 2.39 & 0.03 & 0.17 & 0.16 & 0.67 \\
\hline Average & 19.30 & 78.87 & 10.09 & 0.13 & 2.38 & 0.04 & 0.17 & 0.16 & 0.52 \\
\hline
\end{tabular}

In this study, the wireline logs of the examined wells were first quantitatively investigated to separate the gas-bearing intervals from water zones within Abu Madi sandstones in El-Qara Field.

The gas zone within the El-Qara- 2 well was observed between depths of 3315 and $3358 \mathrm{~m}$. Alternatively, in ElQara-3 well, the appreciative interval was found between depths of 3358 and $3371 \mathrm{~m}$.

The qualitative assessment of the wireline logs showed that the gas zones in each well exhibited the following characteristics (Figs. 3, 4).

1 Constant caliper curve (approximately 8 inches), denoting good borehole conditions and permeability.

2 Low gamma ray values ( $<30 \mathrm{API}$ ), reflecting the low shale content.

3 High values of deep resistivity $\log s$, which probably indicates the presence of non-conducive hydrocarbons (gas).

4 Crossover shape between the neutron porosity and bulk density logs (i.e., the deflection of neutron $\log$ to the right and the density curve to the left), indicating the presence of the sandstone matrix and/or hydrocarbon or water saturating fluids.

Thus, the motivating gas zones in the examined wells were quantitatively evaluated. This evaluation involves calculating the most significant petrophysical parameters necessary for estimating the quality of potential hydrocarbon reservoirs. These parameters include shale volume, total porosity, effective porosity, and water and hydrocarbon saturation (Tables 1,2 ).

\section{Shale volume $\left(V_{s h}\right)$}

The calculated shale volume in the examined zones is shown in track six in Figs. 3 and 4. It is calculated using the gamma ray log according to the following equation (Asquith and Gibson, 1982).

$V_{\mathrm{sh}}=\frac{\left(\mathrm{GR}-\mathrm{GR}_{\min }\right)}{\left(\mathrm{GR}_{\max }-\mathrm{GR}_{\max }\right)}$

where $V_{\text {sh }}$ represents the shale volume, GR represents the gamma ray reading value, $\mathrm{GR}_{\min }$ represents the lowest gamma ray value, and $\mathrm{GR}_{\max }$ represents the maximum gamma ray value.

\section{Total porosity $\left(\phi_{T}\right)$}

Total porosity is determined using the neutron-density logs and is shown in track seven in Figs. 3 and 4. It can be estimated using the following formula (Asquith and Gibson 1982):

$\phi_{\mathrm{T}}=\frac{\phi_{\mathrm{N}}+\phi_{\mathrm{D}}}{2}$

where $\phi_{\mathrm{T}}$ is the total porosity, $\phi_{\mathrm{N}}$ is the neutron porosity, and $\phi_{\mathrm{D}}$ is the density porosity.

\section{Effective porosity $\left(\phi_{\mathrm{e}}\right)$}

The effective porosity curves (track eight in Figs. 3, 4) can be calculated using the following equation (Asquith and Gibson 1982):

$\phi_{e}=\phi_{T} *\left(1-V_{s h}\right)$

where $\phi_{\mathrm{e}}$ is the effective porosity, $\phi_{\mathrm{T}}$ is the total porosity, and $V_{\mathrm{sh}}$ is the shale volume.

\section{Connate water resistivity $\left(\boldsymbol{R}_{\mathrm{w}}\right)$}

According to Asquith and Gibson (1982), the Humble formula should be used to calculate the Fm. factor $F$ for 
Table 2 Well log data and output results for gas zone in El-Qara-3 well

\begin{tabular}{|c|c|c|c|c|c|c|c|c|c|}
\hline \multicolumn{6}{|c|}{ Input well log data } & \multicolumn{4}{|l|}{ Output results } \\
\hline Depth (ft) & $\begin{array}{l}\text { Gamma } \\
\text { Ray } \\
(\mathrm{API})\end{array}$ & Sonic (US/F) & $\begin{array}{l}\text { Deep } \\
\text { resistivity } \\
(\Omega \mathrm{m} 2 / \mathrm{m})\end{array}$ & $\begin{array}{l}\text { Neutron } \\
\text { porosity }\end{array}$ & Density (G/C3) & Shale volume & Total porosity & $\begin{array}{l}\text { Effective } \\
\text { porosity }\end{array}$ & Water saturation \\
\hline 3358.11 & 26.67 & 81.05 & 7.17 & 0.15 & 2.37 & 0.19 & 0.17 & 0.13 & 0.59 \\
\hline 3358.26 & 29.48 & 81.34 & 7.21 & 0.15 & 2.34 & 0.22 & 0.18 & 0.13 & 0.56 \\
\hline 3358.41 & 29.39 & 82.52 & 7.18 & 0.16 & 2.34 & 0.22 & 0.18 & 0.13 & 0.55 \\
\hline 3358.56 & 32.21 & 83.38 & 7.44 & 0.17 & 2.36 & 0.25 & 0.18 & 0.12 & 0.56 \\
\hline 3358.71 & 32.53 & 82.84 & 7.40 & 0.17 & 2.37 & 0.25 & 0.18 & 0.12 & 0.58 \\
\hline 3358.86 & 31.69 & 83.62 & 7.52 & 0.17 & 2.37 & 0.24 & 0.18 & 0.12 & 0.57 \\
\hline 3359.01 & 29.99 & 84.28 & 7.64 & 0.17 & 2.37 & 0.22 & 0.18 & 0.13 & 0.56 \\
\hline 3359.16 & 28.60 & 84.31 & 7.91 & 0.17 & 2.36 & 0.21 & 0.18 & 0.13 & 0.53 \\
\hline 3359.31 & 29.32 & 85.01 & 8.08 & 0.17 & 2.36 & 0.21 & 0.18 & 0.13 & 0.52 \\
\hline 3359.46 & 30.14 & 86.54 & 8.55 & 0.18 & 2.35 & 0.22 & 0.19 & 0.13 & 0.49 \\
\hline 3359.61 & 30.94 & 86.94 & 8.75 & 0.18 & 2.35 & 0.23 & 0.19 & 0.13 & 0.49 \\
\hline 3359.76 & 31.62 & 84.81 & 8.83 & 0.18 & 2.35 & 0.24 & 0.18 & 0.13 & 0.49 \\
\hline 3359.91 & 32.80 & 83.22 & 9.02 & 0.17 & 2.37 & 0.25 & 0.18 & 0.12 & 0.52 \\
\hline 3360.06 & 34.22 & 82.57 & 8.68 & 0.17 & 2.39 & 0.27 & 0.17 & 0.11 & 0.57 \\
\hline 3360.21 & 34.90 & 81.75 & 9.47 & 0.18 & 2.39 & 0.28 & 0.17 & 0.11 & 0.54 \\
\hline 3360.36 & 34.97 & 83.98 & 10.12 & 0.19 & 2.38 & 0.28 & 0.18 & 0.11 & 0.49 \\
\hline 3360.51 & 33.18 & 85.56 & 10.21 & 0.19 & 2.35 & 0.26 & 0.19 & 0.13 & 0.45 \\
\hline 3360.66 & 31.19 & 85.65 & 12.31 & 0.19 & 2.33 & 0.24 & 0.19 & 0.14 & 0.38 \\
\hline 3360.81 & 28.66 & 85.58 & 11.75 & 0.18 & 2.32 & 0.21 & 0.20 & 0.15 & 0.38 \\
\hline 3360.96 & 25.90 & 84.72 & 11.01 & 0.18 & 2.32 & 0.18 & 0.19 & 0.15 & 0.39 \\
\hline 3361.11 & 25.43 & 84.66 & 11.17 & 0.18 & 2.32 & 0.17 & 0.19 & 0.15 & 0.39 \\
\hline 3361.26 & 25.90 & 84.82 & 12.27 & 0.18 & 2.32 & 0.18 & 0.19 & 0.15 & 0.38 \\
\hline 3361.41 & 27.11 & 84.45 & 12.91 & 0.18 & 2.33 & 0.19 & 0.19 & 0.15 & 0.37 \\
\hline 3361.56 & 27.26 & 84.41 & 12.16 & 0.17 & 2.33 & 0.19 & 0.19 & 0.14 & 0.39 \\
\hline 3361.71 & 26.69 & 84.40 & 12.35 & 0.17 & 2.33 & 0.19 & 0.19 & 0.14 & 0.39 \\
\hline 3361.86 & 26.30 & 83.45 & 12.56 & 0.17 & 2.33 & 0.18 & 0.19 & 0.15 & 0.38 \\
\hline 3362.01 & 26.23 & 82.28 & 17.02 & 0.16 & 2.32 & 0.18 & 0.19 & 0.14 & 0.33 \\
\hline 3362.16 & 26.03 & 81.48 & 17.48 & 0.16 & 2.32 & 0.18 & 0.18 & 0.14 & 0.33 \\
\hline 3362.31 & 25.54 & 80.97 & 12.93 & 0.16 & 2.32 & 0.17 & 0.18 & 0.14 & 0.39 \\
\hline 3362.46 & 24.91 & 80.96 & 12.80 & 0.16 & 2.33 & 0.17 & 0.18 & 0.15 & 0.39 \\
\hline 3362.61 & 24.36 & 79.91 & 12.51 & 0.16 & 2.34 & 0.16 & 0.18 & 0.14 & 0.40 \\
\hline 3362.76 & 23.95 & 78.20 & 12.44 & 0.14 & 2.35 & 0.15 & 0.17 & 0.13 & 0.43 \\
\hline 3362.91 & 24.51 & 77.93 & 12.13 & 0.13 & 2.37 & 0.16 & 0.16 & 0.12 & 0.46 \\
\hline 3363.06 & 27.29 & 78.25 & 11.68 & 0.14 & 2.38 & 0.19 & 0.16 & 0.12 & 0.49 \\
\hline 3363.21 & 29.48 & 78.84 & 11.26 & 0.15 & 2.38 & 0.22 & 0.16 & 0.11 & 0.49 \\
\hline 3363.36 & 30.21 & 79.62 & 10.75 & 0.16 & 2.37 & 0.22 & 0.17 & 0.12 & 0.48 \\
\hline 3363.51 & 26.61 & 79.36 & 10.31 & 0.16 & 2.37 & 0.18 & 0.17 & 0.13 & 0.48 \\
\hline 3363.66 & 24.09 & 79.09 & 10.39 & 0.16 & 2.38 & 0.16 & 0.17 & 0.13 & 0.48 \\
\hline 3363.81 & 22.13 & 79.77 & 10.72 & 0.16 & 2.40 & 0.13 & 0.16 & 0.13 & 0.49 \\
\hline 3363.96 & 22.90 & 79.90 & 10.91 & 0.16 & 2.41 & 0.14 & 0.16 & 0.13 & 0.50 \\
\hline 3364.11 & 23.11 & 80.57 & 11.12 & 0.16 & 2.39 & 0.15 & 0.16 & 0.13 & 0.48 \\
\hline 3364.26 & 24.32 & 80.14 & 11.15 & 0.16 & 2.37 & 0.16 & 0.17 & 0.14 & 0.45 \\
\hline 3364.41 & 23.50 & 79.35 & 11.43 & 0.15 & 2.36 & 0.15 & 0.17 & 0.14 & 0.45 \\
\hline 3364.56 & 23.75 & 78.97 & 11.80 & 0.15 & 2.37 & 0.15 & 0.16 & 0.13 & 0.46 \\
\hline 3364.71 & 23.48 & 79.22 & 12.35 & 0.15 & 2.39 & 0.15 & 0.16 & 0.13 & 0.46 \\
\hline 3364.86 & 25.00 & 79.46 & 12.74 & 0.15 & 2.38 & 0.17 & 0.16 & 0.12 & 0.45 \\
\hline 3365.01 & 23.91 & 80.06 & 15.09 & 0.15 & 2.36 & 0.15 & 0.17 & 0.13 & 0.39 \\
\hline
\end{tabular}


Table 2 (continued)

\begin{tabular}{|c|c|c|c|c|c|c|c|c|c|}
\hline \multicolumn{6}{|c|}{ Input well log data } & \multicolumn{4}{|l|}{ Output results } \\
\hline Depth (ft) & $\begin{array}{l}\text { Gamma } \\
\text { Ray } \\
(\mathrm{API})\end{array}$ & Sonic (US/F) & $\begin{array}{l}\text { Deep } \\
\text { resistivity } \\
(\Omega \mathrm{m} 2 / \mathrm{m})\end{array}$ & $\begin{array}{l}\text { Neutron } \\
\text { porosity }\end{array}$ & Density (G/C3) & Shale volume & Total porosity & $\begin{array}{l}\text { Effective } \\
\text { porosity }\end{array}$ & Water saturation \\
\hline 3365.16 & 22.70 & 81.26 & 19.32 & 0.14 & 2.36 & 0.14 & 0.17 & 0.13 & 0.34 \\
\hline 3365.31 & 23.78 & 80.83 & 13.25 & 0.14 & 2.35 & 0.15 & 0.17 & 0.13 & 0.41 \\
\hline 3365.46 & 25.89 & 80.98 & 13.37 & 0.14 & 2.37 & 0.18 & 0.16 & 0.12 & 0.43 \\
\hline 3365.61 & 27.48 & 80.75 & 13.69 & 0.15 & 2.37 & 0.19 & 0.17 & 0.12 & 0.43 \\
\hline 3365.76 & 25.88 & 80.61 & 12.09 & 0.15 & 2.36 & 0.18 & 0.17 & 0.13 & 0.45 \\
\hline 3365.91 & 25.16 & 80.42 & 14.60 & 0.15 & 2.35 & 0.17 & 0.17 & 0.13 & 0.39 \\
\hline 3366.06 & 25.33 & 80.56 & 14.58 & 0.15 & 2.35 & 0.17 & 0.17 & 0.13 & 0.39 \\
\hline 3366.21 & 25.22 & 80.71 & 14.33 & 0.15 & 2.35 & 0.17 & 0.17 & 0.13 & 0.39 \\
\hline 3366.36 & 27.58 & 80.25 & 14.20 & 0.14 & 2.35 & 0.20 & 0.17 & 0.13 & 0.40 \\
\hline 3366.51 & 25.79 & 79.90 & 13.51 & 0.14 & 2.34 & 0.18 & 0.17 & 0.13 & 0.41 \\
\hline 3366.66 & 25.47 & 78.64 & 13.69 & 0.14 & 2.34 & 0.17 & 0.17 & 0.13 & 0.41 \\
\hline 3366.81 & 24.84 & 77.31 & 13.13 & 0.14 & 2.34 & 0.16 & 0.17 & 0.13 & 0.42 \\
\hline 3366.96 & 25.19 & 77.68 & 13.17 & 0.14 & 2.35 & 0.17 & 0.17 & 0.13 & 0.42 \\
\hline 3367.11 & 26.06 & 78.17 & 12.14 & 0.15 & 2.37 & 0.18 & 0.17 & 0.13 & 0.45 \\
\hline 3367.26 & 25.48 & 78.52 & 12.29 & 0.15 & 2.37 & 0.17 & 0.17 & 0.13 & 0.44 \\
\hline 3367.41 & 27.07 & 78.43 & 12.09 & 0.15 & 2.38 & 0.19 & 0.16 & 0.12 & 0.47 \\
\hline 3367.56 & 25.21 & 79.40 & 11.80 & 0.14 & 2.39 & 0.17 & 0.16 & 0.12 & 0.49 \\
\hline 3367.71 & 25.19 & 78.90 & 11.79 & 0.14 & 2.39 & 0.17 & 0.16 & 0.12 & 0.49 \\
\hline 3367.86 & 25.18 & 80.13 & 11.76 & 0.14 & 2.37 & 0.17 & 0.16 & 0.12 & 0.47 \\
\hline 3368.01 & 28.91 & 81.00 & 11.77 & 0.15 & 2.37 & 0.21 & 0.17 & 0.12 & 0.47 \\
\hline 3368.16 & 30.08 & 80.91 & 11.77 & 0.15 & 2.36 & 0.22 & 0.17 & 0.12 & 0.45 \\
\hline 3368.31 & 29.52 & 80.78 & 12.31 & 0.16 & 2.37 & 0.22 & 0.17 & 0.12 & 0.45 \\
\hline 3368.46 & 29.49 & 81.24 & 12.38 & 0.15 & 2.37 & 0.22 & 0.17 & 0.12 & 0.46 \\
\hline 3368.61 & 29.79 & 80.47 & 12.36 & 0.14 & 2.37 & 0.22 & 0.16 & 0.11 & 0.47 \\
\hline 3368.76 & 29.82 & 79.95 & 12.30 & 0.14 & 2.37 & 0.22 & 0.16 & 0.11 & 0.47 \\
\hline 3368.91 & 27.18 & 80.01 & 12.77 & 0.14 & 2.36 & 0.19 & 0.17 & 0.12 & 0.44 \\
\hline 3369.06 & 24.50 & 79.81 & 13.06 & 0.14 & 2.35 & 0.16 & 0.17 & 0.13 & 0.43 \\
\hline 3369.21 & 25.00 & 79.89 & 13.24 & 0.14 & 2.36 & 0.17 & 0.17 & 0.13 & 0.42 \\
\hline 3369.36 & 26.45 & 79.90 & 12.86 & 0.15 & 2.36 & 0.18 & 0.17 & 0.13 & 0.43 \\
\hline 3369.51 & 26.77 & 79.06 & 13.01 & 0.15 & 2.37 & 0.19 & 0.16 & 0.12 & 0.44 \\
\hline 3369.66 & 26.59 & 78.18 & 12.49 & 0.14 & 2.37 & 0.18 & 0.16 & 0.12 & 0.45 \\
\hline 3369.81 & 26.32 & 76.60 & 12.12 & 0.13 & 2.36 & 0.18 & 0.16 & 0.12 & 0.47 \\
\hline 3369.96 & 25.28 & 76.68 & 11.95 & 0.13 & 2.36 & 0.17 & 0.16 & 0.12 & 0.47 \\
\hline 3370.11 & 24.26 & 76.97 & 11.05 & 0.12 & 2.37 & 0.16 & 0.16 & 0.12 & 0.51 \\
\hline 3370.26 & 22.42 & 77.51 & 10.32 & 0.12 & 2.38 & 0.14 & 0.15 & 0.12 & 0.53 \\
\hline 3370.41 & 21.48 & 79.46 & 9.31 & 0.13 & 2.39 & 0.13 & 0.16 & 0.13 & 0.55 \\
\hline 3370.56 & 20.90 & 79.56 & 8.44 & 0.14 & 2.39 & 0.12 & 0.16 & 0.13 & 0.57 \\
\hline 3370.71 & 22.09 & 79.40 & 7.73 & 0.14 & 2.39 & 0.13 & 0.16 & 0.13 & 0.60 \\
\hline 3370.86 & 24.29 & 79.31 & 6.90 & 0.15 & 2.39 & 0.16 & 0.16 & 0.13 & 0.63 \\
\hline 3371.01 & 25.14 & 78.13 & 5.96 & 0.15 & 2.37 & 0.17 & 0.17 & 0.13 & 0.66 \\
\hline 3371.16 & 23.51 & 77.39 & 5.73 & 0.14 & 2.37 & 0.15 & 0.16 & 0.13 & 0.68 \\
\hline Average & 26.78 & 80.86 & 11.40 & 0.15 & 2.36 & 0.19 & 0.17 & 0.13 & 0.46 \\
\hline
\end{tabular}

unconsolidated sands. Because the inspected sands in the examined wells are semifriable to friable, as described in the composite logs, the Humble formula was applied. In this formula, the cementation exponent $m$ is set to 2.15 and the tortuosity factor $a$ is set to 0.62 . Thus, the Archie model (Archie 1942) yields the following equation. 


\section{EI Qara-2 Well}

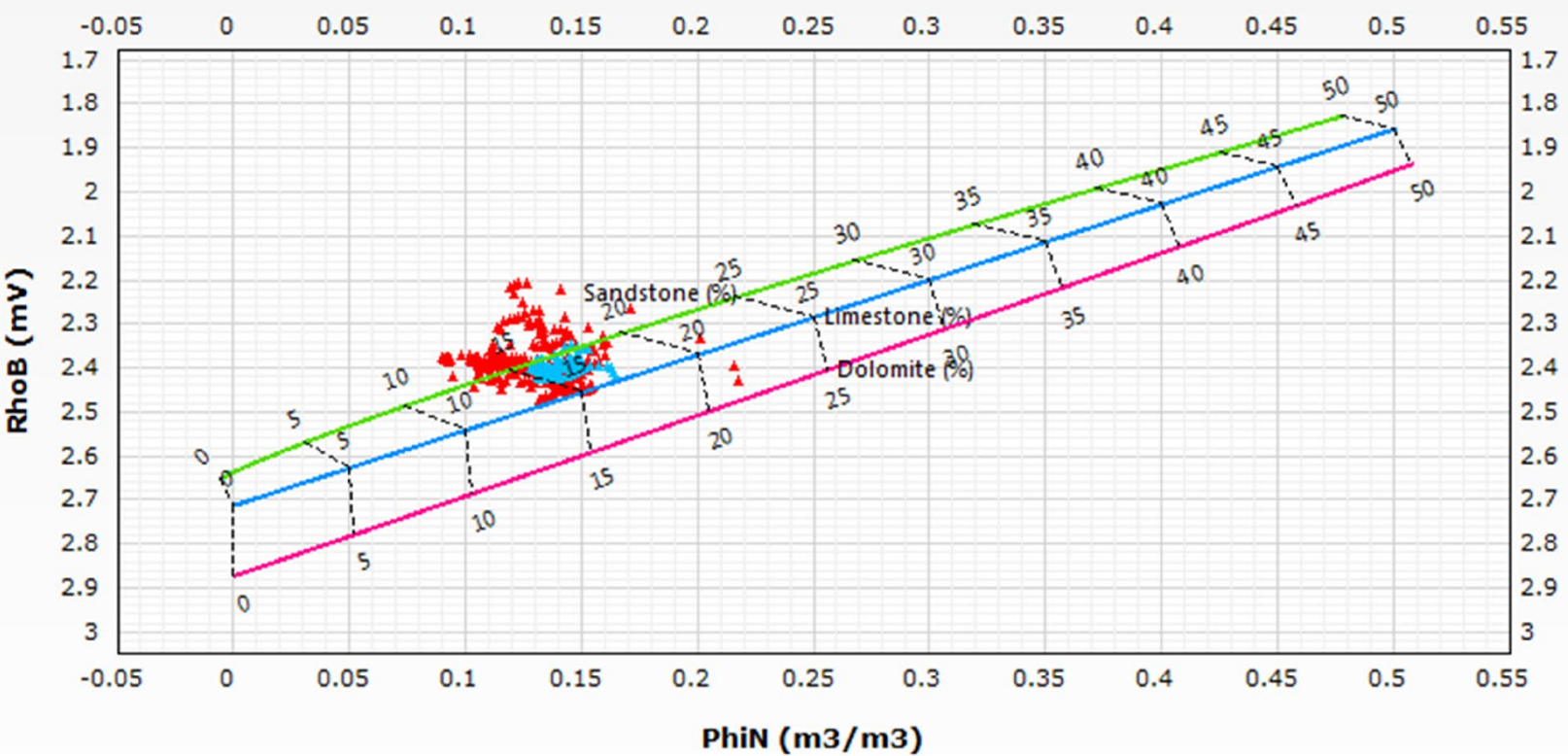

$\square$ Gas zone

$\square$ Water zone

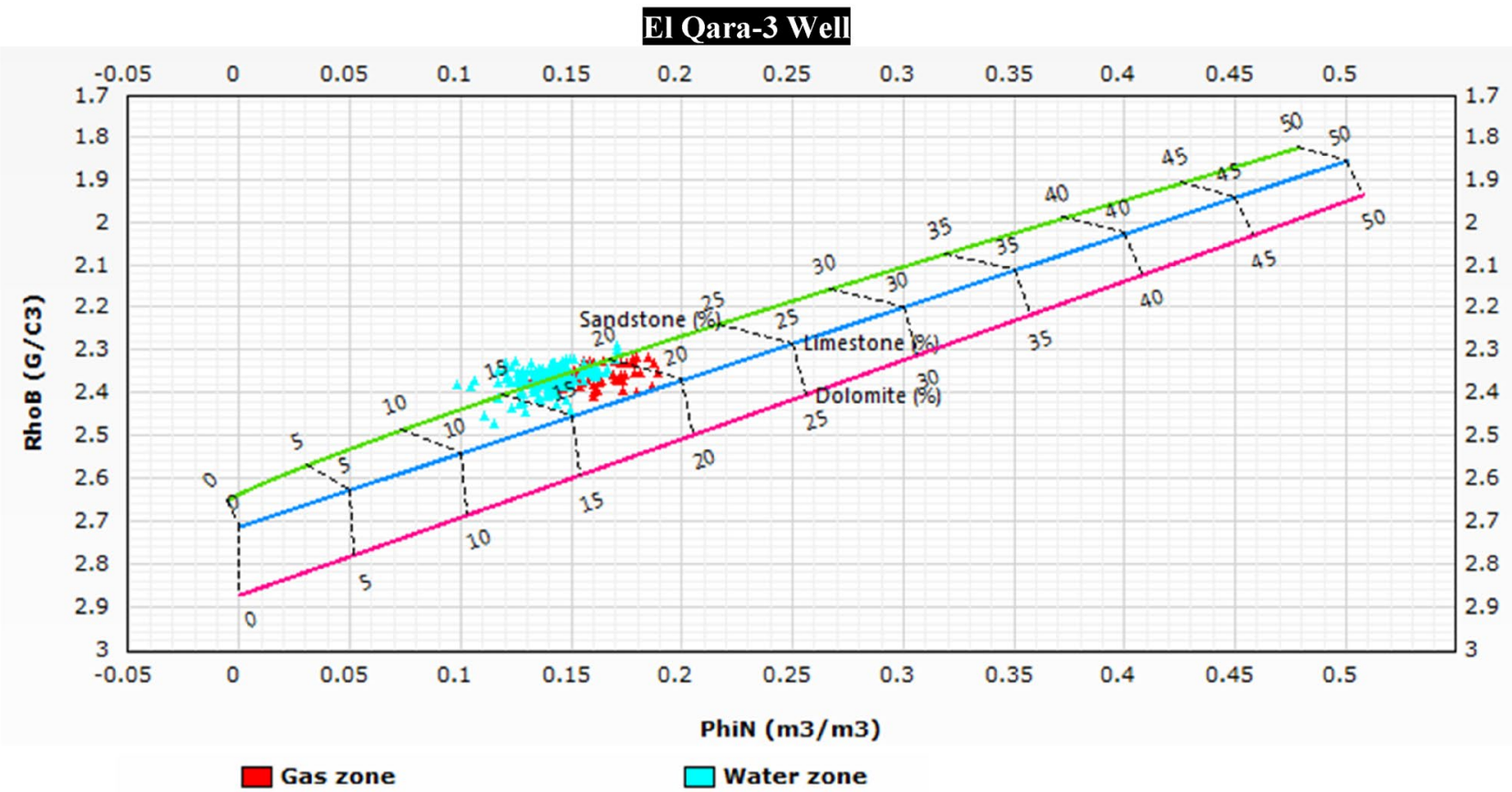

Fig. 5 Density-neutron cross plots (Schlumberger 1972) for the examined zones within Abu Madi Fm. at El-Qara Field. Note that the clustering of plotted points on and near the sandstone line confirms the sandy matrix 

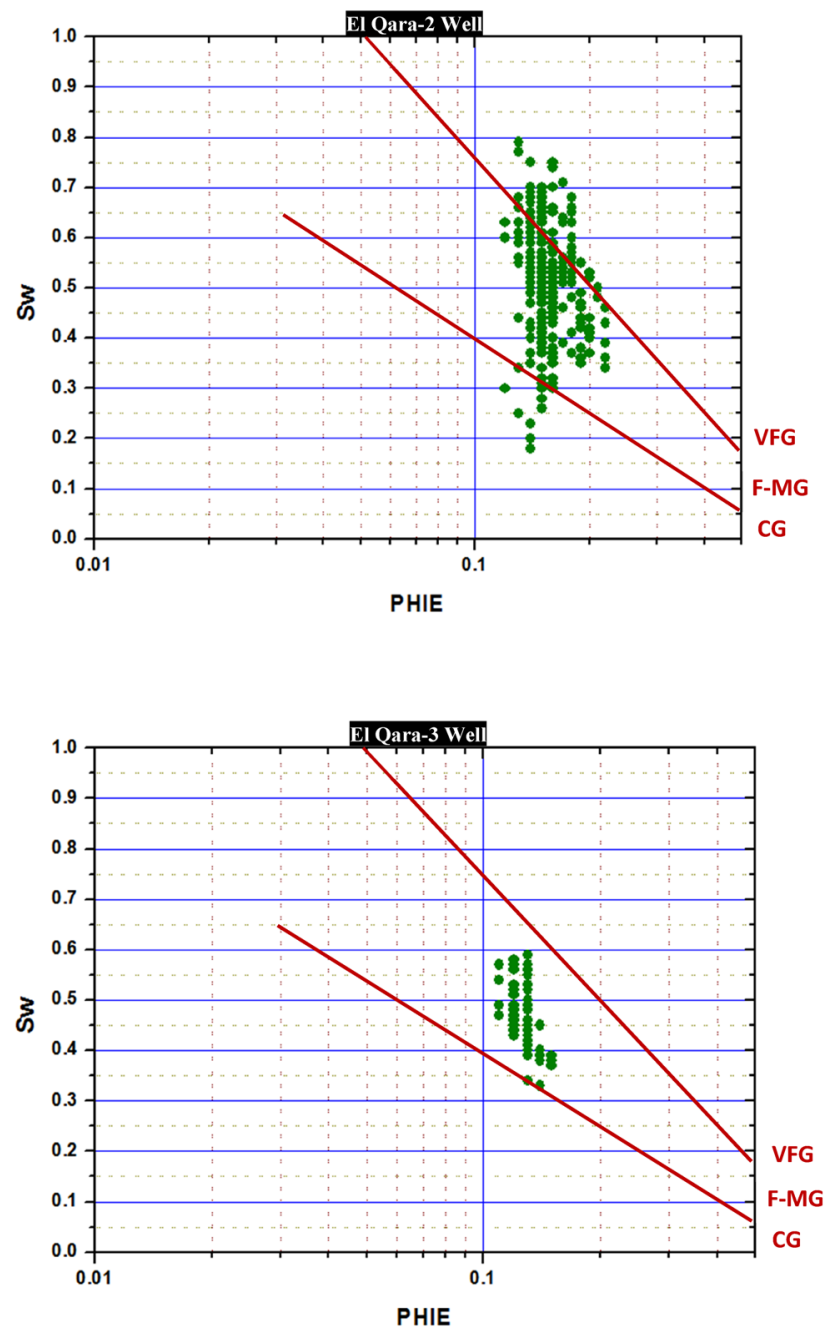

Fig. 6 Water saturation-effective porosity cross plots (Asquith and Gibson 1982) for the examined gas zones. Note that the sand size of the gas zone in El-Qara-2 well differs in size from coarse to very fine, and the sand grains are fine to medium grained in the El-Qara-3 well

$F=a / \phi^{m}$

where $F$ is the Fm. factor, $a$ is the tortuosity factor, $\phi$ is the effective porosity, and $m$ is the cementation exponent.

The average effective porosity in the water zones in both wells was approximately 0.15 , as shown in the water zones of the eighth track in Figs. 3 and 4.

$F=0.62 /(0.15)^{2.15}=36.63$

Moreover, the connate water resistivity $R_{\mathrm{w}}$ can be calculated using the Archie equation (Archie 1942):

$R_{o}=F_{\times} R_{w}$, where $R_{o}$ is the true resistivity $\left(R_{t}\right)$ and $S_{w}=100 \%$. Here, $R_{o}$ is approximately $2.50 \Omega \mathrm{m}^{2} / \mathrm{m}$, as shown in the water zones of the fourth track in Figs. 3 and 4. Thus,

$R_{w}=2.50 / 36.63=0.07 \Omega \mathrm{m}^{2} / \mathrm{m}$.

\section{Water saturation $\left(S_{w}\right)$}

The water saturation in the reservoir zones in both wells is calculated using the Simandoux model (Simandoux 1963):

$\frac{1}{R t}=\frac{\Phi^{m} S w^{n}}{a R w}+\frac{V s h S w}{R s h}$

where $S_{w}$ is the water saturation, $R_{w}$ is the connate water resistivity and is set to $0.07 \Omega \mathrm{m}^{2} / \mathrm{m}, \phi$ is the effective porosity, $V_{s h}$ is the shale volume, $R_{s h}$ is the shale resistivity, and $R_{t}$ is the deep resistivity.

\section{Results and discussion}

The investigation of the constructed neutron-density cross plots (Schlumberger 1972) for the gas and water zones in El-Qara-2 and El-Qara-3 wells showed the clustering of points on and near the sandstone line, confirming the sandy matrix (Fig. 5). The constructed water saturation-effective porosity cross plots (Asquith and Gibson 1982) revealed that the sand grains of the examined zones in El-Qara-2 well varied in size from coarse to very fine-grained. Alternatively, in El-Qara-3 well, most sand grains were fine to medium grained in size (Fig. 6).

The average $V_{s h}$ calculated in the reservoir zone of ElQara-2 well was very low (0.04), while the shale content in the reservoir of El-Qara-3 well increased and reached 0.19 (Tables 1,2). The average total porosity was relatively high and equal in both wells and reached 0.17 (Tables 1,2). The average effective porosity was higher in the El-Qara-2 well (0.16) than in the El-Qara-3 well (0.13) owing to the higher number of shales in the reservoir zone of El-Qara-3 well (Tables 1,2).

The calculated water saturation is shown in blue in track nine in Figs. 3 and 4, while the red color in the same track represents the hydrocarbon saturation. The water saturation was 0.52 in the gas zone of El-Qara-2 well (i.e., hydrocarbon saturation was $48 \%$ ), while water saturation is lower in the reservoir interval of El-Qara-3 well and reached only 0.46 (i.e., hydrocarbon saturation was 54\%) (Tables 1, 2). 


\section{El Qara-2 Well}

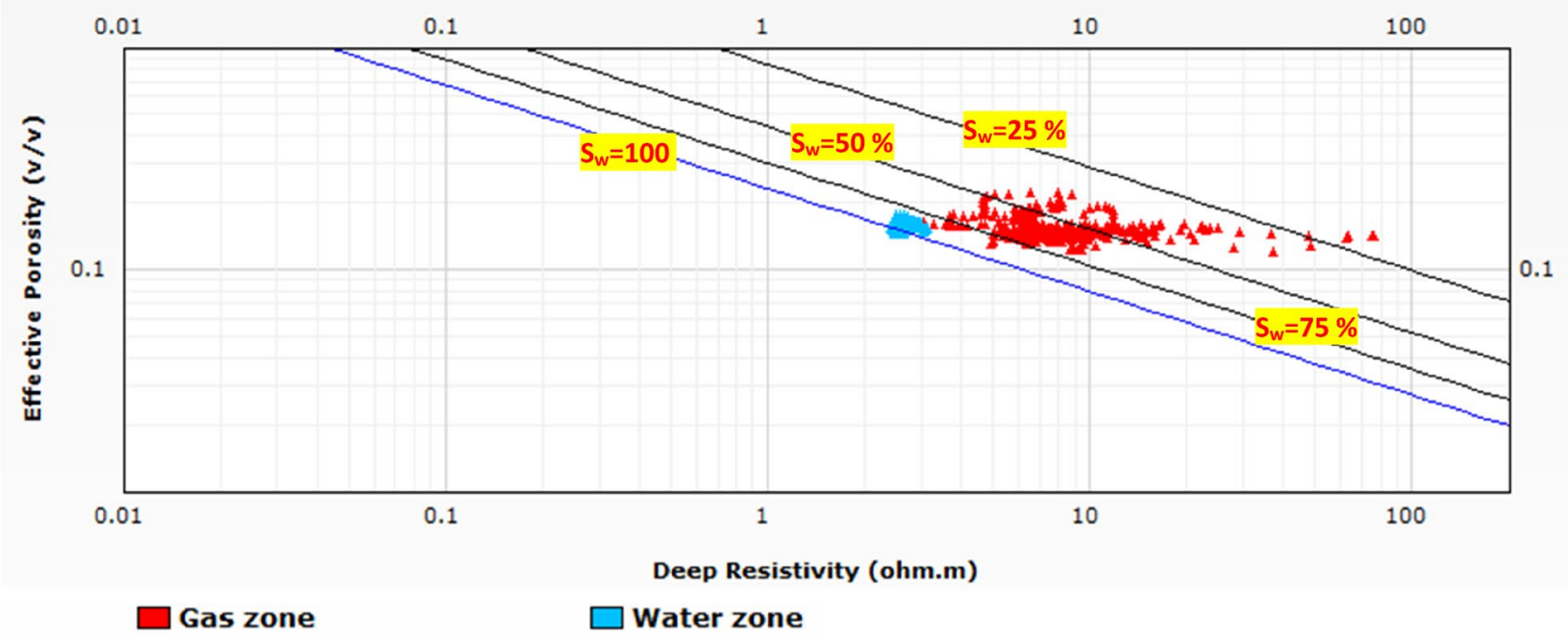

EI Qara-3 Well

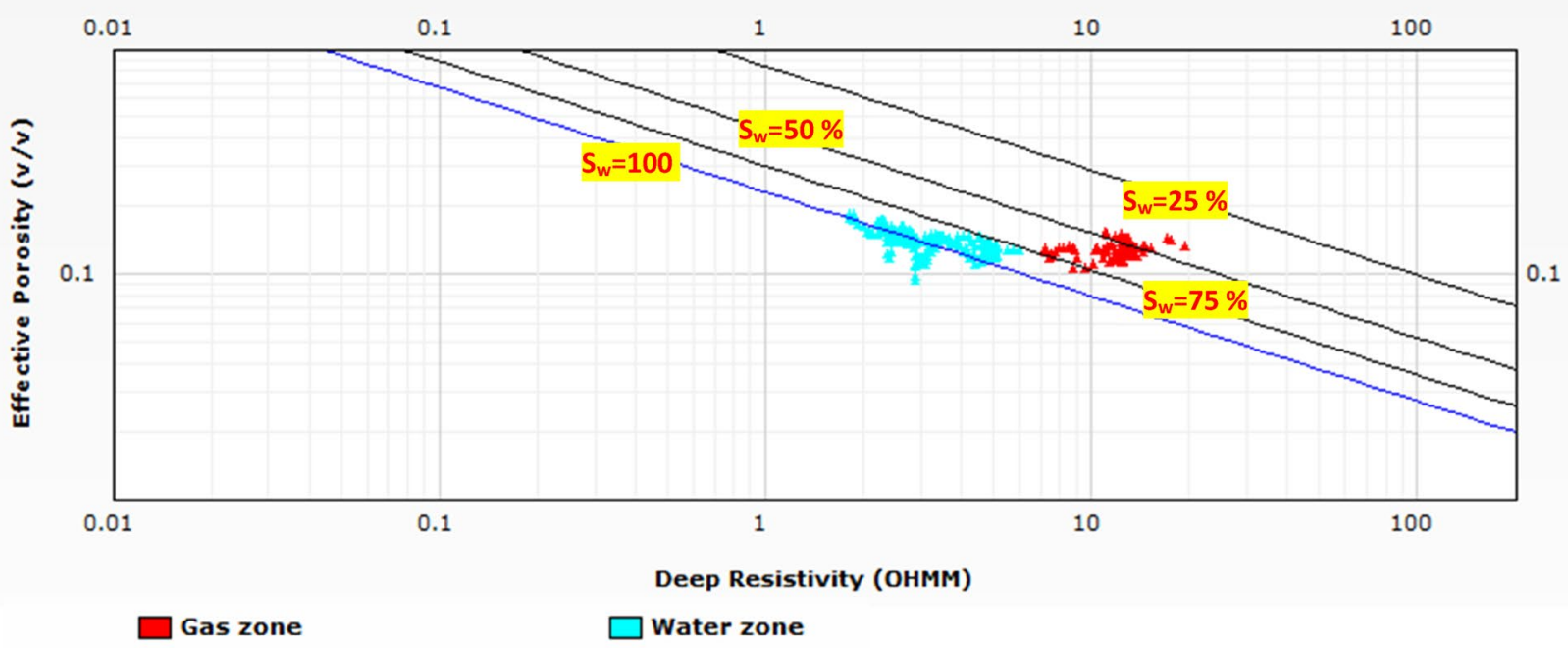

Fig. 7 Pickett plots for the examined zones in El-Qara-2 and El-Qara-3 wells. Note that most points plotted below $S_{W}=75 \%$ line represent the gas-bearing intervals

\section{Pickett plots}

The Pickett cross plots (Pickett 1972) are constructed for the examined zones in Fig. 7. The cross plot presents the relation between the deep resistivity and effective porosity on the $\mathrm{x}$-axis and $\mathrm{y}$-axis, respectively, with logarithmic scales. Points representing the water zones in El-Qara-2 and ElQara-3 wells were clustering around line $S_{w}=100 \%$ (blue). However, most points characterizing the gas zones in the examined wells were clustered and positioned below line $S_{w}=75 \%$, reflecting the gas potentiality of both zones. These 

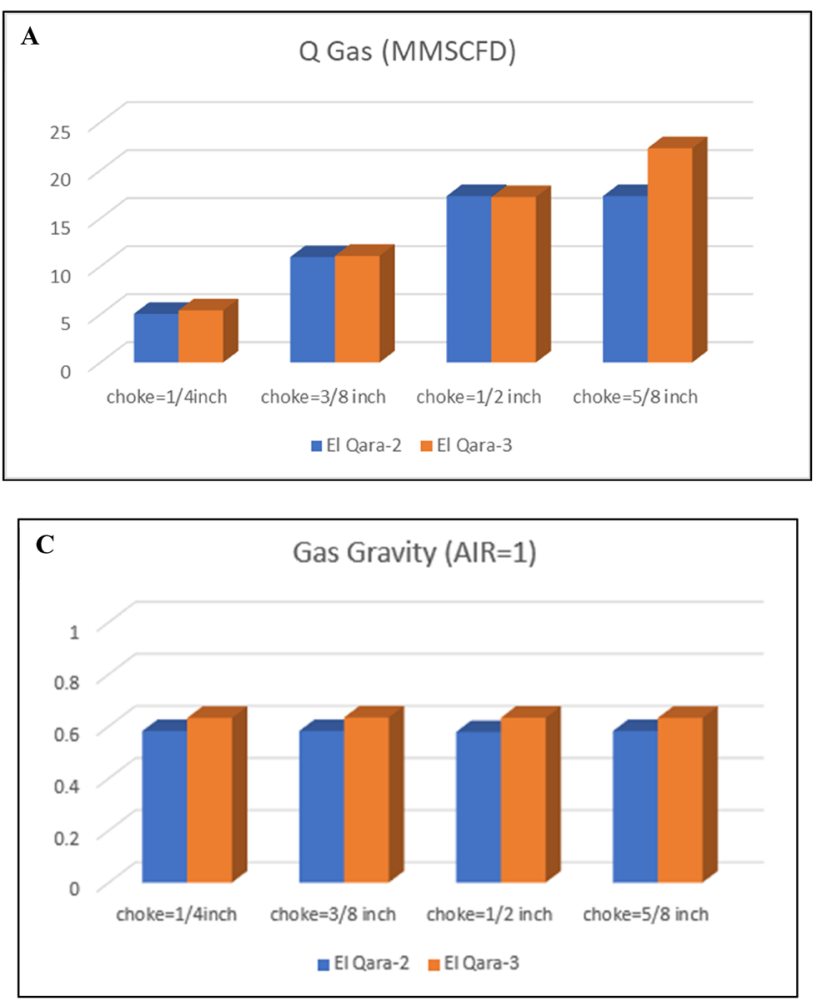
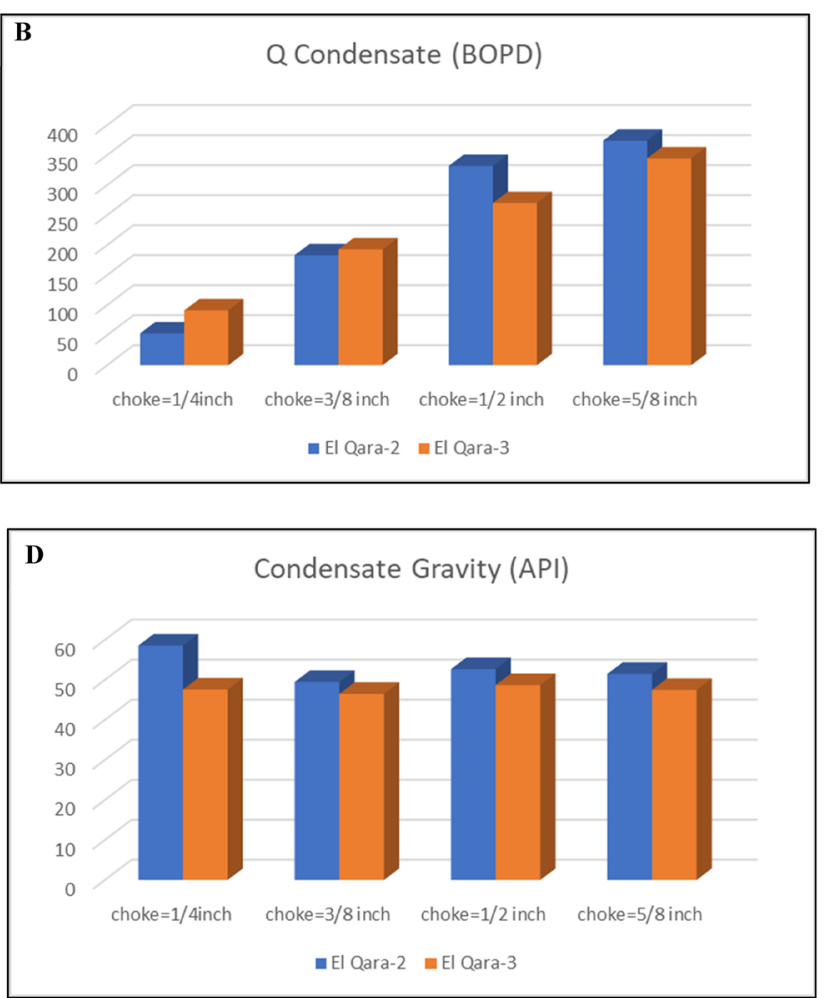

Fig. 8 Bar graphs for the production tests for different choke sizes for the gas zones in the examined wells. a gas rate (Q Gas), b condensate rate (Q Condensate), $\mathbf{c}$ gas gravity, and $\mathbf{d}$ condensate gravity

plots match the results of the calculated water saturation (Tables 1,2), supporting the reliability of mathematical calculations and the significance of the examined zones as potential gas-bearing intervals.

\section{Drill stem test (DST)}

The available DST data for the investigated wells were used to verify the results of this study. DSTs for the water zone in El-Qara-2 well confirmed the production of saltwater, with total dissolved salts $=1024 \mathrm{gm} / \mathrm{L}, \mathrm{NaCl}=26 \mathrm{gm} / \mathrm{L}$, and $\mathrm{pH}=7.9$. Alternatively, the DST results for the water zone in El-Qara-3 well confirmed the production of saltwater, with total dissolved salts $=1060 \mathrm{gm} / \mathrm{L}, \mathrm{NaCl}=16.6$ $\mathrm{gm} / \mathrm{L}$, and $\mathrm{pH}=6.93$.

\section{Production tests}

The production tests for El-Qara-2 and El-Qara-3 wells confirmed the production of gas and condensate from the examined gas zones. The results of different choke sizes $(1 / 4,3 / 8,1 / 2$, and $5 / 8$ inches) for both wells are summarized and shown in Figs. 8 and 9. These results indicate that the characteristics of the promising zones in the two wells were relatively similar, including the produced gas rate ( $\mathrm{Q}$ gas), produced condensate ( $\mathrm{Q}$ condensate), gas gravity, rate condensate gravity, gas-to-oil ratio, flowing tubing head pressure, flowing bottom hole pressure, and static bottom hole pressure. These findings confirm that the two zones are the same hydrocarbon reservoir extending within El-Qara Field, although they are located at different depths. This depth variation is attributed to the presence of normal faults between El-Qara-2 and El-Qara-3 wells (Fig. 10).

The presence of normal faults is abundant and expected in the Late Miocene section beneath the NDB. This is because Abu Madi Fm. is a portion of the Miocene syn-rift megasequence (Sarhan et al. 2014). Figures 11 and 12 show the interpreted seismic sections along the Abu Madi Paleovalley within El-Qara Field. These seismic profiles confirm the existence of growth normal faults associated with syn-rift megasequences. The syn-rift megasequence in the NDB was created because of the Red Sea-Gulf of Suez rift during the Messinian age (Sarhan et al. 2014). Alternatively, the postrift megasequence within the northern-central part of the Nile Delta was formed during the Pliocene-Quaternary age (El-Fawal et al. 2016). 

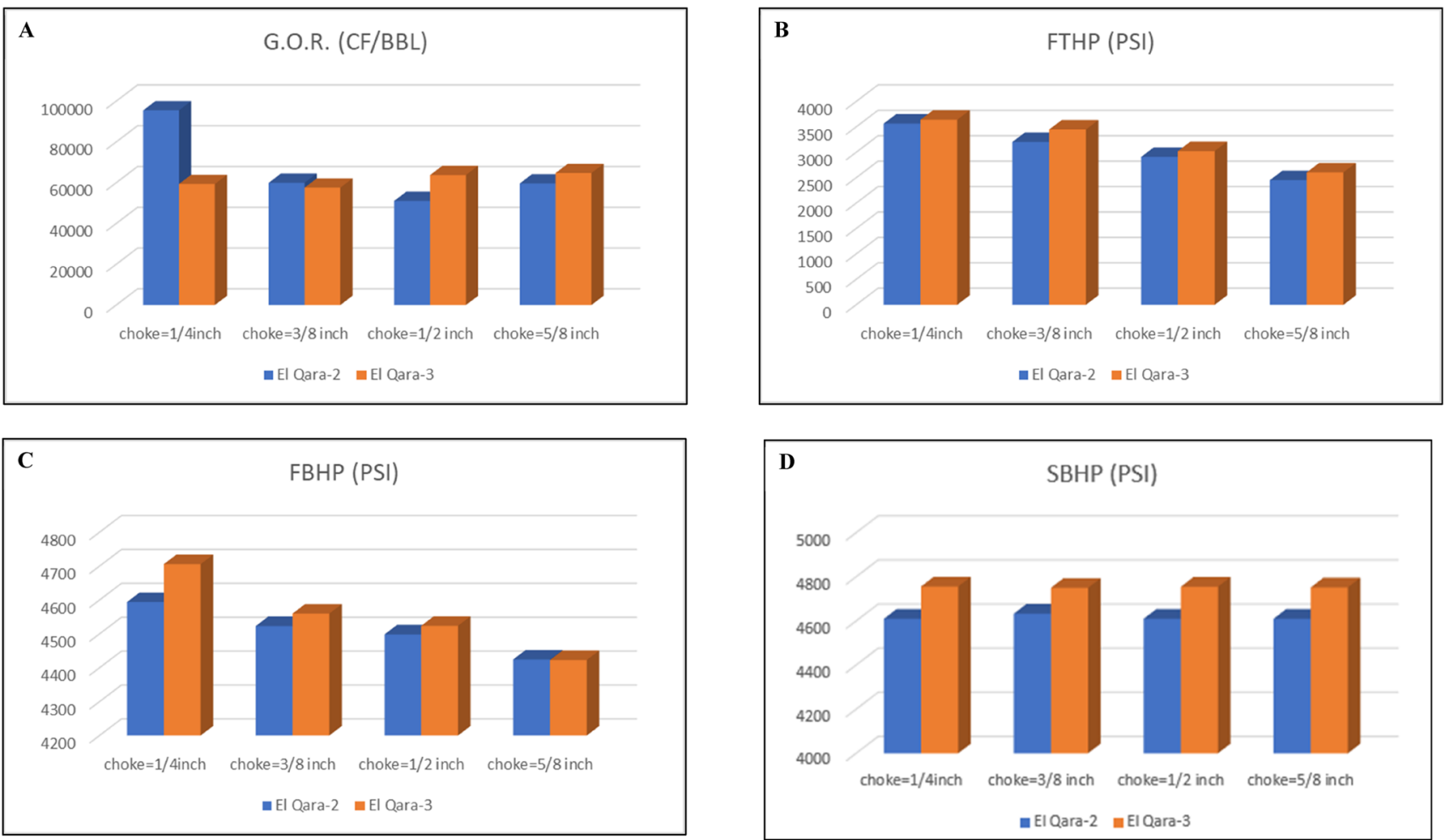

Fig. 9 Bar graphs for the production tests for different choke sizes for the gas zones in the examined wells. a gas-to-oil ratio (G.O.R.), b flowing tubing head pressure (FTHP), $\mathbf{c}$ flowing bottom hole pressure (FBHP), and $\mathbf{d}$ static bottom hole pressure (SBHP)

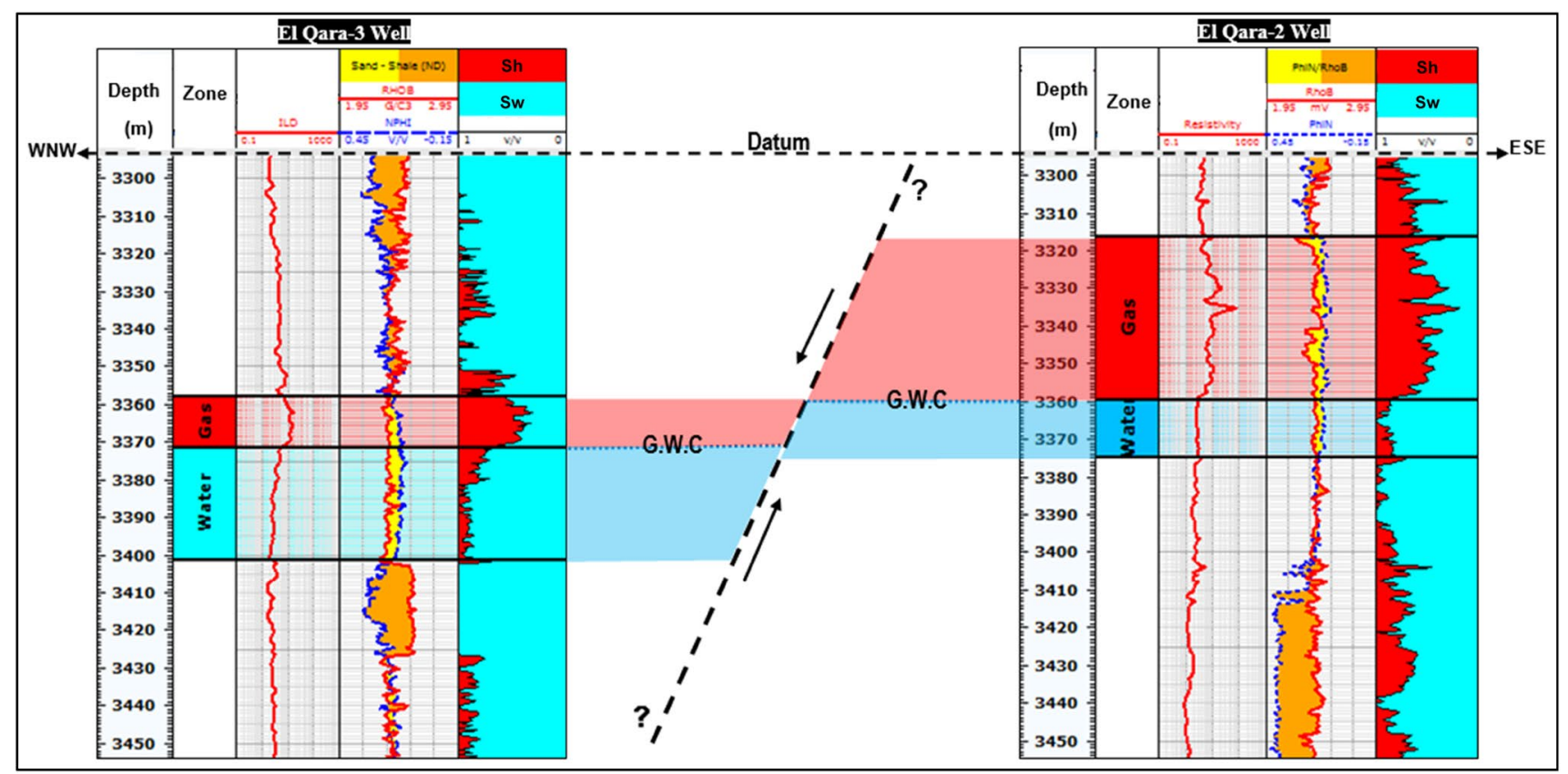

Fig. 10 Schematic cross-section shows the interpreted normal fault, which separates the gas-bearing reservoir within the Abu Madi Fm. between El-Qara-2 and El-Qara-3 wells (refer to Fig. 1b for well locations) 


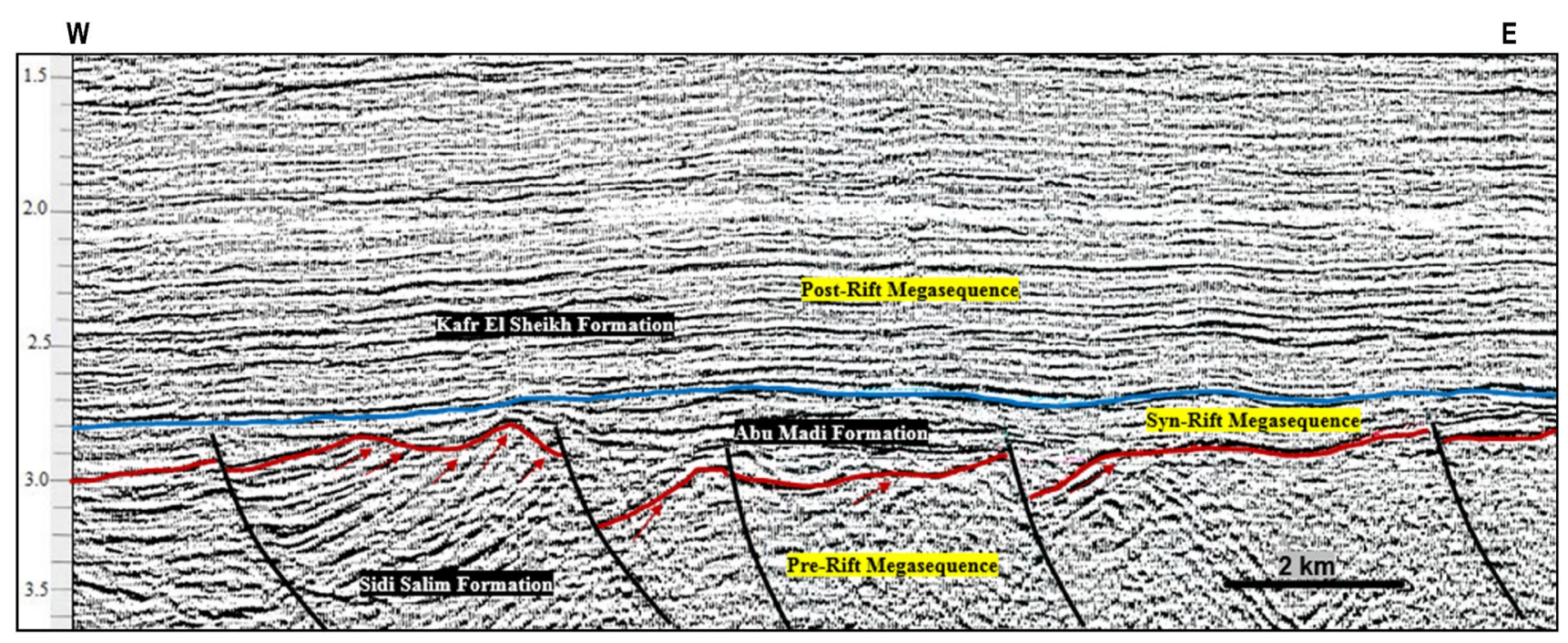

Fig. 11 East-west interpreted seismic profile No. MAN 478-81 shows the toplapping of the base of the syn-rift megasequence to the lower reflectors of the pre-rift megasequence. The abundance of growth normal faults within the syn-rift megasequence, including Abu Madi Fm. (refer to Fig. 1b for seismic line location)

\section{W}

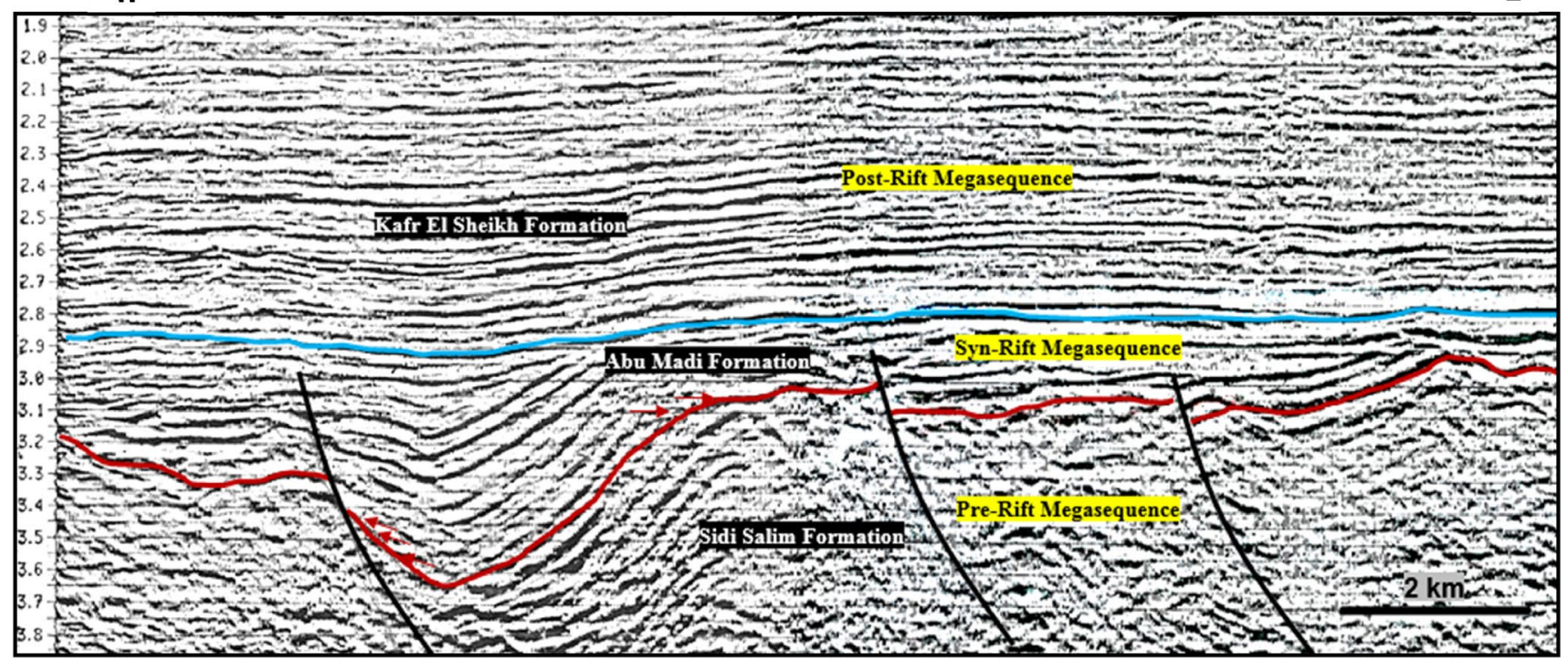

Fig. 12 East-west interpreted seismic section No. Bil 824-85 (modified after Sarhan 2015) displays the onlapping of the internal reflectors of the syn-rift megasequence to the base reflector (refer to Fig. $1 \mathrm{~b}$ for seismic line location)

The deposition of Abu Madi Fm. represents the final stage of the Late Miocene syn-rift megasequence in the NDB (Sarhan et al. 2014). Abu Madi Fm. was strongly affected by tectonic impacts instead of relative sea-level changes (Sarhan 2015). Thus, the facies and distributions of Abu Madi Fm. were controlled by faulting and erosion during the Messinian age (El Heiny et al. 1990).

\section{Conclusions}

The output results of well logging evaluation of the Abu Madi reservoir in El-Qara Field, northern NDB, are listed below. 
- The most promising gas-bearing zone within El-Qara-2 well is located between depths of 3315 and $3358 \mathrm{~m}$, while in El-Qara-3 well, the gas interval is observed between depths of 3358 and $3371 \mathrm{~m}$.

- The calculated petrophysical parameters along with the production tests for both gas intervals reveal relatively similar results, although they are located at different depths. This depth variation is attributed to the presence of normal faults between El-Qara-2 and El-Qara-3 wells.

- The deposition of Abu Madi Fm. was accompanied by the unstable tectonic setting characterized by the presence of active extensional faults between the examined wells in El-Qara Field.

Acknowledgements I am grateful to the Egyptian General Petroleum Corporation (EGPC) and Belayim Petroleum Company (PETROBEL) for releasing the geophysical data presented in this paper. I am much gratitude to the Egyptian Knowledge Bank (EKB) for editing the English language of this paper by Enago services.

\section{Declaration}

Conflict of interest The author declare that have no conflict of interest.

Ethical statements Hereby, I assure that this paper has not been previously published and the manuscript reflects my own research and analysis in a truthful and complete manner.

Open Access This article is licensed under a Creative Commons Attribution 4.0 International License, which permits use, sharing, adaptation, distribution and reproduction in any medium or format, as long as you give appropriate credit to the original author(s) and the source, provide a link to the Creative Commons licence, and indicate if changes were made. The images or other third party material in this article are included in the article's Creative Commons licence, unless indicated otherwise in a credit line to the material. If material is not included in the article's Creative Commons licence and your intended use is not permitted by statutory regulation or exceeds the permitted use, you will need to obtain permission directly from the copyright holder. To view a copy of this licence, visit http://creativecommons.org/licenses/by/4.0/.

\section{References}

Abu El-Ella R (1990) The neogene-quatemary section in the Nile Delta, Egypt; Geology and hydrocarbon potential. J Pet Geol 13:329-340

Archie GE (1942) The electrical resistivity log as an aid in determining some reservoir characteristics. Petrol Technol 5:54-62

Arisi Rota F, Palmieri G, Quagliaroli F (1994) Nile Delta Basin: geological and structural setting. IEOC internal report.

Asquith G, Gibson C (1982) Basic well log analysis for geologists: methods in Exploration series. AAPG, Tulsa, Oklahoma

Barber PM (1981) Messinian subaerial erosion of the Proto-Nile Delta. Mar Geol 44:253-272

Bertello F, Barsoum K, Dalla S, Guessarian S (1996)Temsah discovery: a giant gas field in a deep sea turbidite environment, 13th EGPe Petroleum Conf Explor Cairo, Egypt,1, pp 165-180
Dolson JC, Shann MV, Matbouly S, Harwood C, Rashed R, Hammouda H (2001) AAPG Memoir 74, Chapter 23: The Petroleum Potential of Egypt

Egyptian General Petroleum Corporation (EGPC) (1994) Nile Delta and North Sinai: Fields, Discoveries and Hydrocarbon Potential (a Comprehensive Overview). Egypt, Cairo

EI-Heiny I, Enani N (1996) Regional stratigraphic interpretation of Neogene sediments, Northern Nile Delta. In: 13th EGPC Petroleum Conference on Explor Cairo Egypt, vol 1, pp 270-290

EI-Heiny I, Morsi S (1992) Stratigraphic correlation of Neogene sediments in the eastern Nile Delta and Gulf of Suez. 11th Exploration and Production Conf Cairo, vol 1, pp 166-193

El Heiny I, Rizk R, Hassan M (1990) Sedimentological model for Abu Madi reservoir sands, Abu Madi Field, Nile Delta, Egypt. In 10th Pet. Exploration and Production Conference, vol 2, pp 515-551.

El-Barkooky AN, Helal MA (2002) Sequence stratigraphy and sedimentary history of the Neogene Nile Delta, AAPG Annual International Meeting Cairo, Egypt, pp 27-30

El-Fawal FM, Sarhan MA, Collier REL, Basal A, Abdel Aal MHA (2016) Sequence stratigraphic evolution of the post-rift mega sequence in the northern part of the Nile Delta basin. Egt Arab J Geosci 9(11):1-20

Harms JC, Wary JL (1990) Nile Delta. In: Said R (ed) The Geology of Egypt. Balkema, Rotterdam, pp 329-344

Kamel H, Eita T, Sarhan M (1998) Nile Delta hydrocarbon potentiality. In: Proceedings of 14th EGCP Petrol conference, Egypt, Cairo, pp 485-503

May PR (1991) The Eastern Mediterranean Mesozoic Basin: evolution and oil habitat. AAPG Bull 75:1215-1232

Mosconi A, Rebora A, Venturino G, Bocc P, Khalil M (1996) EgyptNile Delta and North Sinai Cenozoic tectonic evolutionary model: a proposal. In: 13th EGPC Petroleum Conference Explor Cairo, Egypt, vol 1, pp 203-223

Pickett GR (1972) Practical Fm. evaluation. Golden, Colorado, G.R. Pickett, Inc.

Rizzini A, Vezzani F, Cococcetta V, Khalil M (1976): Stratigraphy and sedimentation of the Neogene-Quaternary section in the Nile Delta area. A.R.E. 5th Expl Seminar, Cairo, pp 327-348.

Ross DA, Uchupi E (1977) Structure and sedimentary history of the southeastern Mediterranean-Nile cone area. Am Assoc Petrol Geol Bull 61:872-902

Said R (1962) The geology of Egypt. Elsevier, New York, p 377

Said R (1981) The geologic evolution of the River Nile. SpringerVerlag, New York, p 151

Said R (1990) Geology of Egypt. Balkema, Rotterdam, p 734

Salem AM, Ketzer JM, Morad S, Rizk RR, Al-Aasm IS (2005) Diagenesis and reservoir-quality evolution of incised valley sandstones: evidence from the Abu Madi gas reservoirs (Upper Miocene), the Nile Delta Basin, Egypt. J Sed Res 75:572-584

Sarhan MA (2015) High resolution sequence stratigraphic analysis of the Late Miocene Abu Madi Fm., Northern Nile Delta Basin. NRIAG J Astron Geophys 4(2):298-306

Sarhan MA, Collier REL, Basal A, Aal MHA (2014) Late Miocene normal faulting beneath the northern Nile Delta: NNW propagation of the Gulf of Suez Rift. Arab J Geosci 7(11):4563-4571

Sarhan M, Hemdan K (1994)North Nile Delta structural setting and trapping mechanism, Egypt. In: 12th EGPC Petroleum Conf Explor, Cairo, Egypt, vol 1, pp 1-18

Schlumberger (1972) Log interpretation/charts. Schlumberger Well Services Inc, Houston

Sestini G (1989) Nile Delta: a review of depositional environments and geological history. Geol Soc Lond (special Publications) 41(1):99-127

Simandoux P (1963) Dielectric measurements on porous media, application to the measurements of water saturation: study of behavior 
of argillaceous Fm.s. Revue De L'institut Francais Du Petrol 18(suppl):93-215

Vandre C, Cramer B, Gerling P, Winsemann J (2007) Natural gas Fm in the western Nile delta (Eastern Mediterranean): Thermogenic versus microbial. Organ Geochem 38:523-539
Zaghloul ZM, Shaaban F, Yossef A (2001) Mesozoic and Cenozoic sedimentary basins of the Nile Delta, Egypt. In: Zaghloul ZM, Elgamal M (eds) Deltas modem and ancient. Proc. Mansoura Univ. 1st Internat. Symp. on Deltas, Cairo, Egypt, 21-33.

Publisher's Note Springer Nature remains neutral with regard to jurisdictional claims in published maps and institutional affiliations. 